\title{
Sharp Estimates of Lower Bounds of Polynomial Decay Order of Eigenfunctions
}

\author{
Dedicated to Professor Teruo Ikebe on his sixtieth birthday
}

By

JUN UCHIYAMA* and OSANOBU YAMADA**

\section{§0. Introduction}

In this paper we shall study the lower bounds of polynomial decay order as $|x| \rightarrow \infty$ of the not identically vanishing solution $u(x) \in H_{l o c}^{2}(\Omega)$ of the second order elliptic equation in $\Omega=\left\{x \in \mathbf{R}^{n}|| x \mid>R_{0}\right\}$

$$
-\sum_{i, j=1}^{n}\left(\frac{\partial}{\partial x_{i}}+\sqrt{-1} b_{i}(x)\right) a_{i j}(x)\left(\frac{\partial}{\partial x_{j}}+\sqrt{-1} b_{j}(x)\right) u(x)+\left(q_{1}(x)+q_{2}(x)\right) u(x)=0,
$$

where the matrix $\left(a_{i j}(x)\right)$ is uniformly positive definite, $b_{i}(x)(1 \leq i \leq n)$ and $q_{1}(x)$ are real-valued functions, and $q_{2}(x)$ is a complex-valued function. Our aim is to combine the results given in Uchiyama [3], Yamada [4] and Agmon [1] in one theorem. We shall state the main parts of the assumptions for the case $a_{i j}(x)$ $=\delta_{i j}$ (Kronecker's delta) as follows: there exist some constants $\beta, \gamma_{1}, \gamma_{2}$ and realvalued bounded functions $\sigma(r), \eta(r)$ such that

$$
\begin{aligned}
\beta>0, \quad \gamma_{1}<2, \quad 2-2 \beta<\gamma_{2}<2, & \\
\sigma(r)>0, \quad \lim _{r \rightarrow \infty} \eta(r)=0, & \\
\limsup _{r \rightarrow \infty} r^{2-2 \beta} \sigma(r)^{-1}\left[r \partial_{r} q_{1}(x)\right. & +\left(\gamma_{1}+\eta(r)\right) q_{1}(x)+\sigma(r)^{-1}\left|r q_{2}(x)\right|^{2} \\
& \left.+\left(2-\gamma_{1}\right)^{-1}|B(x) x|^{2}\right]<0, \\
\limsup _{r \rightarrow \infty} r^{2-2 \beta} \sigma(r)^{-1}\left[r \partial_{r} q_{1}(x)\right. & \left.+\left(\gamma_{2}+\eta(r)\right) q_{1}(x)+\left(2-\gamma_{2}\right)^{-1}|B(x) x|^{2}\right]<0,
\end{aligned}
$$

Communicated by S. Matsuura, October 22, 1987.

* Kyoto Institute of Technology, Matsugasaki, Sakyo-ku, Kyoto 606, Japan.

** Department of Mathematics and physics, Faculty of Science and Engineering, Ritsumeikan University, Kyoto 603, Japan. 


$$
q_{2}(x)=O\left(r^{\beta-2} \sigma(r)\right),|B(x) x|=O\left(r^{\beta-1} \sqrt{\sigma(r)}\right) \quad \text { as } r \rightarrow \infty,
$$

where $B(x)=\left(\partial_{i} b_{j}(x)-\partial_{j} b_{i}(x)\right)$ is an $n \times n$ matrix. Moreover we assume that there exist some constants $0<a<1, C>0,-\infty<\delta_{1}<\infty$ and $\delta_{2} \leq \beta-2$ such that

$$
\begin{aligned}
& \int_{\Omega}\left(q_{1}\right)_{-}(x)|w(x)|^{2} d x \leq a \int_{\Omega}|\nabla w(x)|^{2} d x+C \int_{\Omega} r^{\delta_{1}}|w(x)|^{2} d x \\
& \quad \text { for any } w(x) \in C_{0}^{\infty}(\Omega), \\
& \left(\operatorname{Re}\left[q_{2}\right]\right)_{-}(x) \leq C r^{\delta_{2}} \quad \text { for }|x|>R_{0},
\end{aligned}
$$

where $(f)_{-}(x)=\max \{0,-f(x)\}$ for a real-valued function $f(x)$. More detailed conditions are stated in $\S 1$. Then by Theorem 1.2 given in $\S 1$ we have

$$
\liminf _{R \rightarrow \infty} R^{\left(\gamma_{1} / 2\right)+\max \left\{0, \delta_{1}, \delta_{2}\right\}} \exp \left\{\frac{1}{2} \int_{R_{0}}^{R} \frac{\eta(r)+\sigma(r)}{r} d r\right\} \int_{R<|x|<R+1}|u(x)|^{2} d x>0 .
$$

Moreover if

$$
\int_{R_{0}}^{\infty} R^{-\left(\gamma_{1} / 2\right)-\max \left\{0, \delta_{1}, \delta_{2}\right\}} \exp \left\{-\frac{1}{2} \int_{R_{0}}^{R} \frac{\eta(r)+\sigma(r)}{r} d r\right\} d R=+\infty,
$$

then we have $u(x) \notin L^{2}(\Omega)$.

Roughly speaking the case $\sigma(r) \equiv \varepsilon_{0}>0$ (sufficiently small), $\eta(r) \equiv 0$ and $\gamma_{1}$ $=\gamma_{2}$ corresponds to the result in Uchiyama [3], the case $\sigma(r)=\eta(r)=(\log r)^{-1}$ corresponds to Yamada [4] (but no detailed treatment was given), the case $\sigma(r)$ $=\eta(r)=r^{-\varepsilon_{0}}\left(\varepsilon_{0}>0\right.$ sufficiently small $)$ corresponds to Agmon [1]. Yamada [4] and Agmon [1] assumed $q_{1}(x)<0$ for $r>R_{0}$, but we do not assume this condition in this paper. So our results also can be applied to the atomic-type many body potential (e.g. see Remark 1.4).

We note that the smaller $\gamma_{1}<2$ we choose, the better estimate as lower bound we have. In Example 1.7 we choose $\gamma_{1}=2-2 \beta$ and so we cannot, in general, let $\gamma_{2}=\gamma_{1}$. But in case $q_{1}(x)<0$ for $r>R_{0}$ and $|B(x) x|$ $=o\left(r^{\beta-1} \sqrt{\sigma(r)}\right)$ as $r \rightarrow \infty$ we have only to choose $\gamma_{2}$ to satisfy $2-2 \beta<\gamma_{2}<2$ and $\gamma_{1} \leq \gamma_{2}$, which is the reason that Yamada [4] and Agmon [1] did not assume the condition depending on $\gamma_{2}$.

Example 1.7 and Remark 1.8 show the following: let

$$
\left\{\begin{array}{l}
-\Delta u(x)-\left(r^{\theta}+\lambda\right) u(x)+q_{2}(x) u(x)=0 \quad \text { in } \Omega(\theta>0,-\infty<\lambda<\infty), \\
u(x) \in H_{l o c}^{2}(\Omega), \\
\operatorname{supp}[u] \text { is not a compact set in } \bar{\Omega} .
\end{array}\right.
$$

If 


$$
q_{2}(x)=o\left(r^{(\theta / 2)-1}\right) \quad \text { as } r \longrightarrow \infty
$$

then we have

$$
\begin{aligned}
& \lim _{R \rightarrow \infty} R^{(\theta / 2)+\varepsilon} \int_{R<|x|<R+1}|u(x)|^{2} d x=\infty \quad \text { for any } \varepsilon>0, \\
& u(x) \notin L^{2}(\Omega) \quad \text { for } 0<\theta<2 .
\end{aligned}
$$

If

$$
q_{2}(x)=o\left(r^{(\theta / 2)-1}(\log r)^{-1}\right) \quad \text { as } r \rightarrow \infty,
$$

then we have

$$
\begin{aligned}
& \lim _{R \rightarrow \infty} R^{(\theta / 2)}(\log R)^{\varepsilon} \int_{R<|x|<R+1}|u(x)|^{2} d x=+\infty \quad \text { for any } \varepsilon>0, \\
& u(x) \notin L^{2}(\Omega) \quad \text { for } 0<\theta \leq 2 .
\end{aligned}
$$

If

$$
q_{2}(x)=O\left(r^{(\theta / 2)-1-\varepsilon}\right) \text { as } r \longrightarrow \infty \quad \text { for some } \varepsilon>0,
$$

then we have

$$
\begin{aligned}
& \liminf _{R \rightarrow \infty} R^{(\theta / 2)} \int_{R<|x|<R+1}|u(x)|^{2} d x>0, \\
& u(x) \notin L^{2}(\Omega) \quad \text { for } 0<\theta \leq 2,
\end{aligned}
$$

which is the best possible result. These results show that the more gently $q_{2}(x)$ behaves at infinity, the better estimates as lower bounds we have.

Eastham-Kalf [2] has given fruitful informations and rich references on the problem treated in this paper.

In $\S 1$, the assumptions and main results are explained. We give the proof of Theorem 1 in $\S 2$ and the proof of Theorems 2 and 3 in $\S 3$. The method of proof is similar to the one used in Uchiyama [3] and Eastham-Kalf [2, Theorem 6.3.3].

\section{§1. Assumptions and Main Results}

We list up the notations used here, which are the same as given in Uchiyama [3].

\section{Notations:}

$$
\langle\xi, \eta\rangle=\xi_{1} \eta_{1}+\cdots+\xi_{n} \eta_{n} \text { for } \xi=\left(\xi_{1}, \ldots, \xi_{n}\right), \eta=\left(\eta_{1}, \ldots, \eta_{n}\right) \in \mathbb{C}^{n}
$$


$|\xi|=(\langle\xi, \bar{\xi}\rangle)^{1 / 2}$ for $\xi \in \mathbb{C}^{n}$

$\hat{x}=x /|x|$ and $r=|x|$ for $x=\left(x_{1}, \ldots, x_{n}\right) \in \mathbb{R}^{n}$;

$\partial_{j}=\partial / \partial x_{j}$ and $\partial_{r}=\partial / \partial r$;

$D_{j}=\partial_{j}+\sqrt{-1} b_{j}(x)$ and $D=\left(D_{1}, \ldots, D_{n}\right)$;

$f^{\prime}(r)=(d / d r) f(r)$ and $f^{\prime \prime}(r)=\left(d^{2} / d r^{2}\right) f(r)$;

$\nabla f=\left(\partial_{1} f, \ldots, \partial_{n} f\right)$ for a scalar-valued function $f(x)$;

$\operatorname{div} g=\partial_{1} g_{1}+\cdots+\partial_{n} g_{n}$ for a vector-valued function $g(x)=\left(g_{1}(x), \ldots, g_{n}(x)\right)$;

$A=A(x)=\left(a_{i j}(x)\right)$ is an $n \times n$ matrix ;

$B=B(x)=\operatorname{curl} b(x)=\left(\partial_{i} b_{j}(x)-\partial_{j} b_{i}(x)\right)$ is an $n \times n$ matrix ;

$(f)_{-}(x)=\max \{0,-f(x)\} \geq 0$ for a real-valued function $f(x)$;

$\operatorname{supp}[f]$ denotes the closure of $\{x \mid f(x) \neq 0\}$;

$C^{j}(\Omega)$ denotes the class of $j$-times continuously differentiable functions;

$C_{0}^{j}(\Omega)=\left\{f(x) \in C^{j}(\Omega) \mid \operatorname{supp}[f]\right.$ is a compact set in $\left.\Omega\right\}$;

$C_{0}^{\infty}(\Omega)=\bigcap_{j=1}^{\infty} C_{0}^{j}(\Omega)$;

$L^{2}(\Omega)=\left\{\left.f(x)\left|\int_{\Omega}\right| f(x)\right|^{2} d x<\infty\right\}$

$L_{\text {loc }}^{2}(\Omega)=\left\{f(x) \mid\right.$ for any compact set $\left.K \subset \Omega, \int_{K}|f(x)|^{2} d x<\infty\right\}$;

$H^{m}(\Omega)$ denotes the class of $L^{2}$-functions in $\Omega$ such that all distribution derivatives up to $m$ belong to $L^{2}(\Omega)$;

$H_{l o c}^{m}(\Omega)$ denotes the class of $L_{l o c}^{2}$-functions in $\Omega$ such that all distribution derivatives up to $m$ belong to $L_{l o c}^{2}(\Omega)$;

$\left(\int_{|x|=t}-\int_{|x|=s}\right) f(x) d S=\int_{|x|=t} f(x) d S-\int_{|x|=s} f(x) d S$.

Next we shall state the conditions required in the theorems.

\section{Assumptions:}

(A1) each $a_{i j}(x) \in \mathbb{C}^{2}(\Omega)$ is a real-valued function;

(A2) $a_{i j}(x)=a_{j i}(x)$;

(A3) there exists some constant $C_{1} \geq 1$ such that for any $x \in \Omega$ and any $\xi \in \mathbb{C}^{n}$ we have

$$
C_{1}^{-1}|\xi|^{2} \leq\langle A(x) \xi, \bar{\xi}\rangle \leq C_{1}|\xi|^{2}
$$

(B1) each $b_{i}(x)$ is a real-valued function;

(B2) for any $w(x) \in H_{l o c}^{1}(\Omega)$ we have $b_{i}(x) w(x),\left(\partial_{i} b_{j}(x)\right) w(x) \in L_{l o c}^{2}(\Omega)$;

(C1) $q_{1}(x)$ is a real-valued function;

(C2) for any $w(x) \in H_{l o c}^{1}(\Omega)$ we have $\sqrt{\left|q_{1}(x)\right|} w(x) \in L_{l o c}^{2}(\Omega)$;

(C3) for any $w(x) \in H_{l o c}^{1}(\Omega)$ we have $\sqrt{\left|\nabla q_{1}(x)\right|} w(x) \in L_{l o c}^{2}(\Omega)$;

(D1) $q_{2}(x)$ is a complex-valued function; 
(D2) for any $w(x) \in H_{l o c}^{1}(\Omega)$ we have $\sqrt{\left|q_{2}(x)\right|} w(x) \in L_{l o c}^{2}(\Omega)$;

(E) there exists some constant $R_{0}>1$ such that $\Omega \supset\left\{x \in R^{n}|| x \mid>R_{0}\right\}$

(F) there exist some constants $\alpha, \beta, \gamma_{1}, \gamma_{2}, a_{1}, a_{2}, a_{3}, a_{4}$ and some realvalued functions $\eta(r), \sigma(r) \in C^{1}\left(R_{0}, \infty\right)$ such that the following (F1) (F9) hold.

(F1) $0<\alpha<\beta, a_{1}>1, a_{2}>1, a_{3}>0, a_{4}>1$;

(F2) $\sigma(r)=O(1)$ as $r \rightarrow \infty$ and $\sigma(r)>0$ for $r>R_{0}$, $\eta(r)=O(1)$ as $r \rightarrow \infty$,

$\gamma_{1}+\limsup _{r \rightarrow \infty} \eta(r)<2$,

$(2-2 \beta<) 2-2 \alpha<\gamma_{2}+\liminf _{r \rightarrow \infty} \eta(r) \leq \gamma_{2}+\limsup _{r \rightarrow \infty} \eta(r)<2 ;$

(F3) $\lim _{r \rightarrow \infty} r^{\beta-\alpha} \sigma(r)=\infty$;

(F4) $\lim _{r \rightarrow \infty} r^{1-\beta} \sigma(r)^{-1} \sigma^{\prime}(r)=0$ and $\lim _{r \rightarrow \infty} r^{1-\beta} \sigma(r)^{-1} \eta^{\prime}(r)=0$;

(F5) $\limsup _{r \rightarrow \infty} r^{2-2 \beta} \sigma(r)^{-1}\left[r\langle A(x) \hat{x}, \hat{x}\rangle^{-1}\left\langle A(x) \nabla q_{1}(x), \hat{x}\right\rangle\right.$

$$
\begin{aligned}
r \rightarrow \infty & \left(\gamma_{1}+\eta(r)\right) q_{1}(x)+a_{1} \sigma(r)^{-1}\langle A(x) \hat{x}, \hat{x}\rangle^{-1}\left|r q_{2}(x)\right|^{2} \\
+ & a_{2}\left(2-\gamma_{1}-\eta(r)\right)^{-1}\langle A(x) \hat{x}, \hat{x}\rangle^{-2} \\
& \cdot\langle A(x) B(x) A(x) x, B(x) A(x) x\rangle]<0 ;
\end{aligned}
$$

(F6) $\limsup _{r \rightarrow \infty} r^{2-2 \beta} \sigma(r)^{-1}\left[r\langle A(x) \hat{x}, \hat{x}\rangle^{-1}\left\langle A(x) \nabla q_{1}(x), \hat{x}\right\rangle\right.$

$$
\begin{aligned}
& +\left(\gamma_{2}+\eta(r)\right) q_{1}(x)+a_{3} \sigma(r)^{-1}\langle A(x) \hat{x}, \hat{x}\rangle^{-1}\left|r q_{2}(x)\right|^{2} \\
& +a_{4}\left(2-\gamma_{2}-\eta(r)\right)^{-1}\langle A(x) \hat{x}, \hat{x}\rangle^{-2} \\
& \quad \cdot\langle A(x) B(x) A(x) x, B(x) A(x) x\rangle]<0
\end{aligned}
$$

(F7) $\lim _{r \rightarrow \infty} \sigma(r)^{-1}\left(a_{i j}(x)-\delta_{i j}\right)=0$, where $\delta_{i j}$ is the kronecker's delta;

(F8) $\lim _{r \rightarrow \infty} r \sigma(r)^{-1} \partial_{k} a_{i j}(x)=0$;

(F9) $\lim _{r \rightarrow \infty} r^{2-\beta} \sigma(r)^{-1} \partial_{k} \partial_{l} a_{i j}(x)=0$;

(G1) there exist some constants $0<a_{5}<1,-\infty<\delta_{1}<\infty$ and $C_{2}>0$ such that for any $w(x) \in C_{0}^{\infty}(\Omega)$ we have

$$
\int_{\Omega}\left(q_{1}\right)_{-}(x)|w(x)|^{2} d x \leq a_{5} \int_{\Omega}|\nabla w(x)|^{2} d x+C_{2} \int_{\Omega} r^{\delta_{1}}|w(x)|^{2} d x ;
$$

(G2) there exist some constants $\delta_{2} \leq \beta-2$ and $C_{3}>0$ such that for any $r>R_{0}$ we have

$$
\left(\operatorname{Re}\left[q_{2}\right]\right)_{-}(x) \leq C_{3} \min \left\{r^{\delta_{2}}, r^{\beta-2} \sigma(r)\right\},
$$

where $\operatorname{Re}[z]$ means the real part of $z \in \mathbf{C}$.

Now we have the

Theorem 1.1. Let $u(x)$ satisfy

$$
(*):\left\{\begin{array}{l}
-\langle D, A D\rangle u(x)+\left\{q_{1}(x)+q_{2}(x)\right\} u(x)=0 \text { in } \Omega, \\
u(x) \in H_{\text {loc }}^{2}(\Omega), \\
\operatorname{supp}[u] \text { is not a compact set in } \bar{\Omega} \text { (closure of } \Omega) .
\end{array}\right.
$$


Let $(A) \sim(G)$ hold. Then we have the following:

(1) $\quad \liminf _{R \rightarrow \infty} R^{\left(\gamma_{1} / 2\right)} \Phi(R) \int_{|x|=R}\left[|\langle A D u, \hat{x}\rangle|^{2}+\left\{r^{-2}+\left(q_{1}\right)_{-}\right\}|u|^{2}\right] d S>0$,

where

$$
\Phi(R)=\exp \left\{\frac{1}{2} \int_{R_{0}}^{R} \frac{\eta(r)+\sigma(r)}{r} d r\right\}
$$

(2) for any $\varepsilon>0$

$$
\liminf _{R \rightarrow \infty} R^{\left(\gamma_{1} / 2\right)+\max \left\{0, \delta_{1}, \delta_{2}\right\}} \Phi(R) \int_{R<|x|<R+\varepsilon}|u|^{2} d x>0 ;
$$

(3) moreover if

$$
\int_{R_{0}}^{\infty} R^{-\left(\gamma_{1} / 2\right)-\max \left\{0, \delta_{1}, \delta_{2}\right\}} \Phi(R)^{-1} d R=+\infty,
$$

then $u(x) \notin L^{2}(\Omega)$.

Now we shall consider the more special case $a_{i j}(x)=\delta_{i j}$ under the weaker conditions.

Theorem 1.2. Let $u(x)$ satisfy

$$
(* *):\left\{\begin{array}{l}
-\langle D, D\rangle u(x)+\left\{q_{1}(x)+q_{2}(x)\right\} u(x)=0 \text { in } \Omega, \\
u(x) \in H_{l o c}^{2}(\Omega), \\
\operatorname{supp}[u] \text { is not a compact set in } \bar{\Omega} .
\end{array}\right.
$$

We assume $(\mathrm{B}) \sim(\mathrm{G})$ with $a_{i j}(x)=\delta_{i j}$ except for $(\mathrm{C} 3)$. Instead of $(\mathrm{C} 3)$ we assume

(C3)' for any $w(x) \in H_{\text {loc }}^{1}(\Omega)$ we have $\left|\partial_{r} q_{1}(x)\right|^{1 / 2} w(x) \in L_{\text {loc }}^{2}(\Omega)$.

Then we have the same results as given in Theorem $1(1) \sim(3)$, where we replace $a_{i j}(x)$ with $\delta_{i j}$.

Lastly we shall consider the most special case $a_{i j}(x)=\delta_{i j}$ and $b_{i}(x)=0$ under the weakest conditions.

Theorem 1.3. Let $u(x)$ satisfy

$$
(* * *):\left\{\begin{array}{l}
-\Delta u(x)+\left\{q_{1}(x)+q_{2}(x)\right\} u(x)=0 \text { in } \Omega, \\
u(x) \in H_{\text {loc }}^{2}(\Omega), \\
\operatorname{supp}[u] \text { is not a compact set in } \bar{\Omega},
\end{array}\right.
$$

where $\Delta$ is a Laplacian in $\mathbb{R}^{n}$. We assume $(\mathrm{C}) \sim(\mathrm{G})$ with $a_{i j}(x)=\delta_{i j}$ and $b_{i}(x)$ $=0$ except for $(\mathrm{C} 3)$ and $(\mathrm{F} 2)$. Instead of $(\mathrm{C} 3)$ and $(\mathrm{F} 2)$ we assume $(\mathrm{C} 3)^{\prime}$ and 
$(\mathrm{F} 2)^{\prime} \sigma(r)=O(1)$ as $r \rightarrow \infty$ and $\sigma(r)>0$ for $r>R_{0}$,

$\eta(r)=O(1)$ as $r \rightarrow \infty$,

$\gamma_{i}+\eta(r) \leq 2$ for $i=1,2$ and $r>R_{0}$,

$(2-2 \beta<) 2-2 \alpha<\gamma_{2}+\liminf _{r \rightarrow \infty} \eta(r)$.

Then we have the same results as given in Theorem $1(1) \sim(3)$, where we replace $a_{i j}(x)$ with $\delta_{i j}$ and $b_{i}(x)$ with 0 .

Remark 1.4. We have the following:

(1) If $\eta(r)=0$ and $\sigma(r)=v>0$ where $v$ is a constant, then $\lim _{R \rightarrow \infty} R^{-v / 2} \Phi(R)<\infty$.

This case is the one considered in Uchiyama [3].

(2) If $\eta(r)=\sigma(r)=(\log r)^{-1}$, then $\lim _{R \rightarrow \infty}(\log R)^{-1} \Phi(R)<\infty$. This case almost meets with Yamada [4]. However [4] did not give its complete proof and assumed more strict conditions such as $q_{1}(x)<0$ for $r>R_{0}$.

(3) If there exists $\lim _{R \rightarrow \infty} \Phi(R)$, the results given in Agmon [1] are almost reproduced. However [1] assumed more strict conditions such as $q_{1}(x)<0$ for $r>R_{0}$.

(4) In our assumptions that $q_{1}(x)<0$ for $r>R_{0}$ is not assumed. Then we can apply our theorem to the atomic type many body potential

$$
q_{1}(x)=-\sum_{i=1}^{N} \frac{z_{i}}{\left|x^{(i)}\right|}+\sum_{1 \leq i<j \leq N} \frac{z_{i j}}{\left|x^{(i)}-x^{(j)}\right|}-\lambda,
$$

where $x=\left(x^{(1)}, \ldots, x^{(N)}\right) \in \mathbf{R}^{3 N}, x^{(i)}=\left(x_{3 i-2}, x_{3 i-1}, x_{3 i}\right) \in \mathbf{R}^{3}, \lambda>0, z_{i}$ and $z_{i j}$ are real constants. In this case we choose $\gamma_{1}=\gamma_{2}=1$ and $\eta(r)=0$.

Remark 1.5. If we add the following conditions

$$
\begin{array}{ll}
q_{2}(x)=O\left(r^{\beta-2} \sigma(r)\right) & \text { as } r \rightarrow \infty, \\
|B A x|=O\left(r^{\beta-1} \sqrt{\sigma(r)}\right) & \text { as } r \longrightarrow \infty
\end{array}
$$

in $(F)$, then (F5) and (F6) can be replaced with weaker conditions

$$
\begin{aligned}
& \limsup _{r \rightarrow \infty} r^{2-2 \beta} \sigma(r)^{-1}\left[r\langle A \hat{x}, \hat{x}\rangle^{-1}\left\langle A \nabla q_{1}, \hat{x}\right\rangle+\left(\gamma_{1}+\eta(r)\right) q_{1}(x)\right. \\
& \quad+\sigma(r)^{-1}\langle A \hat{x}, \hat{x}\rangle^{-1}\left|r q_{2}(x)\right|^{2} \\
& \left.\quad+\left(2-\gamma_{1}-\eta(r)\right)^{-1}\langle A \hat{x}, \hat{x}\rangle^{-2}\langle A B A x, B A x\rangle\right]<0
\end{aligned}
$$

$(\mathrm{F} 6)^{\prime}$

$$
\begin{aligned}
& \limsup _{r \rightarrow \infty} r^{2-2 \beta} \sigma(r)^{-1}\left[r\langle A \hat{x}, \hat{x}\rangle^{-1}\left\langle A \nabla q_{1}, \hat{x}\right\rangle+\left(\gamma_{2}+\eta(r)\right) q_{1}(x)\right. \\
& \left.\quad+\left(2-\gamma_{2}-\eta(r)\right)^{-1}\langle A \hat{x}, \hat{x}\rangle^{-2}\langle A B A x, B A x\rangle\right]<0 .
\end{aligned}
$$

In fact the quantity given in (F5) depends continuously on $a_{1}$ and $a_{2}$ under our additional conditions. So (F5)' leads to (F5). The same happens in (F6). 
Remark 1.6. If in (F5)

$$
2-2 \alpha<\gamma_{1}+\liminf _{r \rightarrow \infty} \eta(r) \leq \gamma_{1}+\limsup _{r \rightarrow \infty} \eta(r)<2
$$

holds, then (F6) is automatically satisfied. In fact we have only to choose $\gamma_{2}$ $=\gamma_{1}, a_{3}=a_{1}$ and $a_{4}=a_{2}$ in (F6).

Example 1.7. In $(* *)$ let $n=3$ and

$$
\begin{aligned}
& q_{1}(x)=h(x)+V(x)-\lambda, \\
& q_{2}(x)=o\left(r^{(\theta / 2)-1}\right) \quad \text { as } r \longrightarrow \infty, \\
& b_{1}(x)=-2^{-1} b_{0} x_{2} f(r), b_{2}(x)=2^{-1} b_{0} x_{1} f(r), b_{3}(x)=0,
\end{aligned}
$$

where

$-\infty<\lambda, b_{0}<\infty$ are constants,

$h(x)$ is a negative continuous homogeneous function of degree $\theta>0$,

$V(x)$ is a real-valued function satisfying

$$
V(x)=o\left(r^{\theta}\right), \partial_{r} V(x)=o\left(r^{\theta-1}\right) \quad \text { as } r \longrightarrow \infty,
$$

$f(r) \in C^{1}\left(R_{0}, \infty\right)$ is a real-valued function satisfying

$$
r f^{\prime}(r)+2 f(r)=o\left(r^{(\theta / 2)-1}\right) \quad \text { as } r \longrightarrow \infty .
$$

In this case $(B) \sim(E)$ are satisfied, where we replace $(\mathrm{C} 3)$ with $(\mathrm{C} 3)^{\prime}$. We choose in $(\mathrm{F})$ and $(\mathrm{G})$

$$
\begin{aligned}
& \beta=1+(\theta / 2)>0, \gamma_{1}=-\theta(=2-2 \beta), \eta(r)=\sigma(r), \\
& \delta_{1}=\theta, \delta_{2}=(\theta / 2)-1=\beta-2<\theta=\delta_{1},
\end{aligned}
$$

and let $\alpha, a_{1}, a_{2}, a_{3}, a_{4}$ be arbitrary constants satisfying

$$
0<\alpha<1+(\theta / 2)=\beta, a_{1}>1, a_{2}>1, a_{3}>0, a_{4}>1 .
$$

Then we have

$$
\left(\gamma_{1} / 2\right)+\max \left\{0, \delta_{1}, \delta_{2}\right\}=\theta / 2
$$

Noting

$$
|B x|^{2}=4^{-1} b_{0}^{2}\left(x_{1}^{2}+x_{2}^{2}\right)\left|r f^{\prime}(r)+2 f(r)\right|^{2},
$$

we have

$$
\begin{aligned}
& r^{2-2 \beta} \sigma(r)^{-1}\left[r \partial_{r} q_{1}(x)+\left(\gamma_{1}+\eta(r)\right) q_{1}(x)+a_{1} \sigma(r)^{-1}\left|r q_{2}(x)\right|^{2}\right. \\
& \left.\quad+a_{2}\left(2-\gamma_{1}-\eta(r)\right)^{-1}|B x|^{2}\right]
\end{aligned}
$$




$$
\begin{aligned}
= & r^{-\theta} h(x)+r^{-\theta} \sigma(r)^{-1}\left[r \partial_{r} V(x)+(\sigma(r)-\theta) V(x)\right] \\
- & \lambda r^{-\theta} \sigma(r)^{-1}(\sigma(r)-\theta) \\
+ & a_{1} r^{-\theta} \sigma(r)^{-2}\left|r q_{2}(x)\right|^{2}+a_{2}(2+\theta-\sigma(r))^{-1} 4^{-1} b_{0}^{2} \\
& \times r^{-\theta}\left(x_{1}^{2}+x_{2}^{2}\right) \sigma(r)^{-1}\left|r f^{\prime}(r)+2 f(r)\right|^{2} .
\end{aligned}
$$

Now we shall consider the following three cases.

Case 1. Let

$$
\begin{array}{ll}
V(x)=o\left(r^{\theta}\right), \partial_{r} V(x)=o\left(r^{\theta-1}\right) & \text { as } r \longrightarrow \infty, \\
q_{2}(x)=o\left(r^{(\theta / 2)-1}\right) & \text { as } r \longrightarrow \infty, \\
r f^{\prime}(r)+2 f(r)=o\left(r^{(\theta / 2)-1}\right) & \text { as } r \longrightarrow \infty .
\end{array}
$$

In this case we choose

$$
\sigma(r) \equiv \varepsilon,
$$

where $\varepsilon$ is a constant satisfying $0<\varepsilon<2+\theta$. Noting

$$
r^{-\theta} h(x) \leq \max \{h(x)|| x \mid=1\}<0 \text { for any } r>R_{0},
$$

(F) and (G) are satisfied by any $\gamma_{2} \in(\min \{2-2 \alpha-\varepsilon,-\theta\}, 2-\varepsilon)$. Then by Theorem 1.2 and $\lim _{r \rightarrow \infty} r^{-\varepsilon} \Phi(r)<\infty$, we have for $u(x)$ satisfying $(* *)$

$$
\left\{\begin{array}{l}
\lim _{R \rightarrow \infty} R^{(\theta / 2)+\varepsilon} \int_{R<|x|<R+1}|u(x)|^{2} d x=+\infty \quad \text { for any } \varepsilon>0, \\
u(x) \notin L^{2}(\Omega) \quad \text { for } 0<\theta<2 .
\end{array}\right.
$$

Case 2. Let

$$
\begin{array}{ll}
V(x)=o\left(r^{\theta}(\log r)^{-1}\right), \partial_{r} V(x)=o\left(r^{\theta-1}(\log r)^{-1}\right) & \text { as } r \longrightarrow \infty, \\
q_{2}(x)=o\left(r^{(\theta / 2)-1}(\log r)^{-1}\right) & \text { as } r \longrightarrow \infty, \\
r f^{\prime}(r)+2 f(r)=o\left(r^{(\theta / 2)-1}(\log r)^{-1 / 2}\right) & \text { as } r \longrightarrow \infty .
\end{array}
$$

In this case we choose for any $\varepsilon>0$

$$
\sigma(r)=\varepsilon(\log r)^{-1},
$$

and then (F) and $(\mathrm{G})$ are satisfied by any $\gamma_{2} \in(2-2 \alpha, 2)$. So we have by Theorem 1.2 and $\lim _{r \rightarrow \infty}(\log r)^{-\varepsilon} \Phi(r)<\infty$

$$
\begin{cases}\lim _{R \rightarrow \infty} R^{(\theta / 2)}(\log R)^{\varepsilon} \int_{R<|x|<R+1}|u(x)|^{2} d x=+\infty, & \text { for any } \varepsilon>0, \\ u(x) \notin L^{2}(\Omega) & \text { for } 0<\theta \leq 2 .\end{cases}
$$


Case 3. Let for some $\varepsilon>0$

$$
\begin{array}{ll}
V(x)=O\left(r^{\theta-\varepsilon}\right), \partial_{r} V(x)=O\left(r^{\theta-1-\varepsilon}\right) & \text { as } r \rightarrow \infty, \\
q_{2}(x)=O\left(r^{(\theta / 2)-1-\varepsilon}\right) & \text { as } r \rightarrow \infty, \\
r f^{\prime}(r)+2 f(r)=O\left(r^{(\theta / 2)-1-\varepsilon}\right) & \text { as } r \rightarrow \infty .
\end{array}
$$

In this case we choose

$$
\sigma(r)=r^{-\varepsilon^{\prime}}
$$

where $\varepsilon^{\prime}$ is a constant satisfying $0<\varepsilon^{\prime}<\min \{\varepsilon, \beta-\alpha\}$. Then $(\mathrm{F})$ and $(\mathrm{G})$ are satisfied by any $\gamma_{2} \in(2-2 \alpha, 2)$. So we have by Theorem 1.2 and $\lim _{r \rightarrow \infty} \Phi(r)<\infty$

$$
\left\{\begin{array}{l}
\liminf _{R \rightarrow \infty} R^{(\theta / 2)} \int_{R<|x|<R+1}|u(x)|^{2} d x>0, \\
u(x) \notin L^{2}(\Omega) \quad \text { for } 0<\theta \leq 2 .
\end{array}\right.
$$

Remark 1.8. The result given in Example 1.7 Case 3 is best possible. In fact we shall consider the following case in $(* *)$ :

$$
\begin{aligned}
& q_{1}(x)=-r^{\theta} \quad(\theta>0), \\
& q_{2}(x)=0, \\
& \left(b_{1}(x), \ldots, b_{n}(x)\right)=(0, \ldots, 0) .
\end{aligned}
$$

Then

$$
u_{0}(x)=r^{(2-n) / 2} J_{|n-2| /(2+\theta)}\left(\frac{2}{2+\theta} r^{1+(\theta / 2)}\right)
$$

satisfies $(* *)$ with $\Omega=\mathbb{R}^{n}$, where $J_{v}(r)$ denotes the Bessel function of the first kind of order $v$. This solution $u_{0}(x)$ satisfies

$$
\left\{\begin{array}{l}
\limsup _{R \rightarrow \infty} R^{(\theta / 2)} \int_{R<|x|<R+1}\left|u_{0}(x)\right|^{2} d x<+\infty, \\
u_{0}(x) \in L^{2}(\Omega) \quad \text { for } \theta>2,
\end{array}\right.
$$

since $J_{v}(r)=O\left(r^{-1 / 2}\right)$ as $r \rightarrow \infty$.

\section{§2. Proof of Theorem 1}

In this section all the conditions $(\mathrm{A}) \sim(\mathrm{G})$ are assumed. And let $u(x)$ satisfy $(*)$, which is given in Theorem 1 .

Definition 2.1. For real-valued functions $\rho(r) \in C^{2}\left(R_{0}, \infty\right), f(x) \in C^{1}(\Omega)$ and 
$g(x) \in C^{1}(\Omega)$, let

$$
\begin{aligned}
& v=v(x ; \rho)=e^{\rho(r)} u(x), \\
& k_{1}=k_{1}(x ; \rho)=-\left\{\rho^{\prime}(r)\right\}^{2}\langle A(x) \hat{x}, \hat{x}\rangle, \\
& k_{2}=k_{2}(x ; \rho)=\rho^{\prime \prime}(r)\langle A(x) \hat{x}, \hat{x}\rangle+\rho^{\prime}(r) \operatorname{div}\{A(x) \hat{x}\}, \\
& F(t ; \rho, f, g)=\int_{|x|=t}\left[f ( x ) \langle A \hat { x } , \hat { x } \rangle \left\{2\langle A \hat{x}, \hat{x}\rangle^{-1}|\langle A D v, \hat{x}\rangle|^{2}-\langle A D v, \overline{D v}\rangle\right.\right. \\
& \left.\left.\quad-\left(q_{1}+k_{1}\right)|v|^{2}\right\}+g(x) \operatorname{Re}[\langle A D v, \hat{x}\rangle \bar{v}]\right] d S .
\end{aligned}
$$

Lemma 2.2. We have for $t>R_{0}$

$$
\begin{aligned}
& F(t ; \rho, f, g)=e^{2 \rho(t)} \int_{|x|=t}\left[f(x)\left\{2|\langle A D u, \hat{x}\rangle|^{2}-\langle A \hat{x}, \hat{x}\rangle\langle A D u, \overline{D u}\rangle\right\}\right. \\
& \quad+\left\{2 \rho^{\prime} f\langle A \hat{x}, \hat{x}\rangle+g\right\} \operatorname{Re}[\langle A D u, \hat{x}\rangle \bar{u}] \\
& \left.\quad+\left\{2 f \rho^{\prime 2}\langle A \hat{x}, \hat{x}\rangle^{2}+\left(g \rho^{\prime}-f q_{1}\right)\langle A \hat{x}, \hat{x}\rangle\right\}|u|^{2}\right] d S .
\end{aligned}
$$

Proof. Noting Definition 2.1, we have the assertion by straight-forward calculation.

Lemma 2.3. For any $t>s>R_{0}$ we have

$$
\begin{aligned}
F(t ; & \rho, f, g)-F(s ; \rho, f, g) \\
= & \int_{s<|x|<t}\left[\left\{2\langle A \hat{x}, \hat{x}\rangle \partial_{r} f+g-\langle A \nabla f, \hat{x}\rangle-f \operatorname{div}(A \hat{x})\right\}\langle A \hat{x}, \hat{x}\rangle^{-1}\right. \\
& \cdot|\langle A D v, \hat{x}\rangle|^{2} \\
+ & \left\{2 r^{-1} f\langle A \hat{x}, \hat{x}\rangle+g-\langle A \nabla f, \hat{x}\rangle-f \operatorname{div}(A \hat{x})\right\} \\
& \quad \cdot\left\{\langle A D v, \overline{D v}\rangle-\langle A \hat{x}, \hat{x}\rangle^{-1}|\langle A D v, \hat{x}\rangle|^{2}\right\} \\
+ & 2 \operatorname{Re}\left[\left\langle A D v,\left(\nabla-\hat{x} \partial_{r}\right) f\right\rangle\langle A \overline{D v}, \hat{x}\rangle\right] \\
+ & 2 r^{-1} f\left\{|A D v|^{2}-\langle A \hat{x}, \hat{x}\rangle\langle A D v, \overline{D v}\rangle\right\} \\
+ & 2 f \operatorname{Re}[\langle(\langle A D v, \nabla\rangle A) \overline{D v}, \hat{x}\rangle]-f \operatorname{Re}[\langle(\langle\hat{x}, A \nabla\rangle A) D v, \overline{D v}\rangle] \\
+ & 2 f \operatorname{Re}\left[\sqrt{-1}\left\langle A B A \hat{x},\left(D v-\hat{x}\langle A \hat{x}, \hat{x}\rangle{ }^{-1}\langle A D v, \hat{x}\rangle\right)\right\rangle \bar{v}\right] \\
+ & 2 f \operatorname{Re}\left[\langle A D v, \hat{x}\rangle \overline{q_{2} v}\right]+\operatorname{Re}[\langle A D v, \nabla g\rangle \bar{v}] \\
+ & \left.\left\{(g-\langle A \nabla f, \hat{x}\rangle-f \operatorname{div}(A \hat{x})) q_{1}-f\left\langle A \nabla q_{1}, \hat{x}\right\rangle+g \operatorname{Re}\left[q_{2}\right]\right\}|v|^{2}\right] d x \\
+ & \quad \int_{s<|x|<t}\left[4 \rho^{\prime} f|\langle A D v, \hat{x}\rangle|^{2}+2\left(f k_{2}+g \rho^{\prime}\right) \operatorname{Re}[\langle A D v, \hat{x}\rangle \bar{v}]\right. \\
+ & \left.\left\{(g-\langle A \nabla f, \hat{x}\rangle-f \operatorname{div}(A \hat{x})) k_{1}-f\left\langle A \nabla k_{1}, \hat{x}\right\rangle+g k_{2}\right\}|v|^{2}\right] d x .
\end{aligned}
$$


Proof. See Lemmas 2.7 and 2.8 of Uchiyama [3]. In order to obtain the above relation, the conditions $(\mathrm{A}) \sim(\mathrm{E})$ are fully used.

The meaning of the following Definition 2.4 can be partly clarified by Lemma 2.5 .

Definition 2.4. For $i=1,2$ and $x$ satisfying $r=|x|>R_{0}$, let

$$
\begin{aligned}
f_{i}(x)= & \langle A \hat{x}, \hat{x}\rangle^{-1} r^{\left(\gamma_{t} / 2\right)} \Phi(r), \\
g_{i}(x)= & h_{i}(x) r^{\left(\gamma_{t} / 2\right)-1} \Phi(r), \\
h_{i}(x)= & 2^{-1}\left\{\sigma(r)-\eta(r)-\gamma_{i}\right\}+r\langle A \hat{x}, \hat{x}\rangle^{-1} \operatorname{div}(A \hat{x}) \\
& +r\left\langle A \nabla\left(\langle A \hat{x}, \hat{x}\rangle^{-1}\right), \hat{x}\right\rangle,
\end{aligned}
$$

where

$$
\Phi(r)=\exp \left\{\frac{1}{2} \int_{R_{0}}^{r} \frac{\eta(r)+\sigma(r)}{r} d r\right\} .
$$

And for $w \in H_{l o c}^{2}(\Omega), i=1,2$ and $x$ satisfying $r=|x|>R_{0}$, let

$$
\begin{aligned}
G_{i}(x ; w)= & \sigma(r)\langle A \hat{x}, \hat{x}\rangle^{-1}|\langle A D w, \hat{x}\rangle|^{2} \\
& +\left(2-\gamma_{i}-\eta(r)\right)\left\{\langle A D w, \overline{D w}\rangle-\langle A \hat{x}, \hat{x}\rangle^{-1}|\langle A D w, \hat{x}\rangle|^{2}\right\} \\
& +2 r \operatorname{Re}\left[\left\langle A D w, \nabla\left(\langle A \hat{x}, \hat{x}\rangle^{-1}\right)\right\rangle\langle A \overline{D w}, \hat{x}\rangle\right] \\
& +2\left\{\langle A \hat{x}, \hat{x}\rangle^{-1}|A D w|^{2}-\langle A D w, \overline{D w}\rangle\right\} \\
& +2 r\langle A \hat{x}, \hat{x}\rangle^{-1} \operatorname{Re}[\langle(\langle A D w, \nabla\rangle A) \overline{D w}, \hat{x}\rangle] \\
& -r\langle A \hat{x}, \hat{x}\rangle^{-1} \operatorname{Re}[\langle(\langle\hat{x}, A D\rangle A) D w, \overline{D w}\rangle] \\
& -2\langle A \hat{x}, \hat{x}\rangle^{-1} \operatorname{Re}\left[\sqrt { - 1 } \left\langleA B A x,\left(D w-\hat{x}\langle A \hat{x}, \hat{x}\rangle^{-1}\right.\right.\right. \\
& +\langle A D w, \hat{x}\rangle)\rangle \bar{w}] \\
& +2 r\langle A \hat{x}, \hat{x}\rangle^{-1} \operatorname{Re}\left[\langle A D w, \hat{x}\rangle \overline{q_{2} w}\right]+\operatorname{Re}\left[\left\langle A D w, \nabla h_{i}\right\rangle \bar{w}\right] \\
& +(2 r)^{-1} h_{i}\left\{\eta(r)+\sigma(r)+\gamma_{i}-2\right\} \operatorname{Re}[\langle A D w, \hat{x}\rangle \bar{w}] \\
& -\left\{r\langle A \hat{x}, \hat{x}\rangle^{-1}\left\langle A \nabla q_{1}, \hat{x}\right\rangle+\left(\gamma_{i}+\eta(r)\right) q_{1}-h_{i} \operatorname{Re}\left[q_{2}\right]\right\}|w|^{2} .
\end{aligned}
$$

Lemma 2.5. We have the following relations for $i=1,2$ :

(1) for $r>R_{0}$

$$
\begin{aligned}
& g_{i}-\left\langle A \nabla f_{i}, \hat{x}\right\rangle-f_{i} \operatorname{div}(A \hat{x})=-\left(\gamma_{i}+\eta(r)\right) r^{\left(\gamma_{i} / 2\right)-1} \Phi(r), \\
& 2\langle A \hat{x}, \hat{x}\rangle \partial_{r} f_{i}+g_{i}-\left\langle A \nabla f_{i}, \hat{x}\right\rangle-f_{i} \operatorname{div}(A \hat{x}) \\
& \quad=\left\{\sigma(r)+2 r\langle A \hat{x}, \hat{x}\rangle \partial_{r}\left(\langle A \hat{x}, \hat{x}\rangle^{-1}\right)\right\} r^{\left(\gamma_{z} / 2\right)-1} \Phi(r),
\end{aligned}
$$




$$
2 r^{-1} f_{i}\langle A \hat{x}, \hat{x}\rangle+g_{i}-\left\langle A \nabla f_{i}, \hat{x}\right\rangle-f_{i} \operatorname{div}(A \hat{x})=\left(2-\gamma_{i}-\eta(r)\right) r^{\left(\gamma_{2} / 2\right)-1} \Phi(r),
$$

(2) $F\left(t ; \rho, f_{i}, g_{i}\right)-F\left(s ; \rho, f_{i}, g_{i}\right)$

$$
\begin{gathered}
=\int_{s<|x|<t} r^{\left(\gamma_{t} / 2\right)-1} \Phi(r)\left[G_{i}(x ; v)+4 r \rho^{\prime}\langle A \hat{x}, \hat{x}\rangle^{-1}|\langle A D v, \hat{x}\rangle|^{2}\right. \\
+2\left\{r \rho^{\prime \prime}+r \rho^{\prime}\langle A \hat{x}, \hat{x}\rangle^{-1} \operatorname{div}(A \hat{x})+h_{i} \rho^{\prime}\right\} \operatorname{Re}[\langle A D v, \hat{x}\rangle \bar{v}] \\
+\langle A \hat{x}, \hat{x}\rangle\left\{\left(\gamma_{i}+\eta(r)\right) \rho^{\prime 2}+2 r \rho^{\prime} \rho^{\prime \prime}-r \rho^{\prime 2}\langle A D(\langle A \hat{x}, \hat{x}\rangle-1), \hat{x}\rangle\right. \\
\left.\left.+h_{i} \rho^{\prime \prime}+h_{i} \rho^{\prime}\langle A \hat{x}, \hat{x}\rangle^{-1} \operatorname{div}(A \hat{x})\right\}|v|^{2}\right] d x
\end{gathered}
$$

for $t>s>R_{0}$.

Proof. Noting Definition 2.4, Lemma 2.3 and $\Phi^{\prime}(r)=(2 r)^{-1}\{\sigma(r)$ $+\eta(r)\} \Phi(r)$, we have the assertions by direct calculation.

We prepare an auxiliary Lemma 2.6 .

Lemma 2.6. We have the following:

(1) $\lim _{r \rightarrow \infty} \sigma(r)^{-1}(\langle A \hat{x}, \hat{x}\rangle-1)=0$ and $\lim _{r \rightarrow \infty}\langle A \hat{x}, \hat{x}\rangle=1$,

(2) $\lim _{r \rightarrow \infty} r \sigma(r)^{-1} \nabla(\langle A \hat{x}, \hat{x}\rangle)=0$ and $\lim _{r \rightarrow \infty} r \sigma(r)^{-1} \nabla\left(\langle A \hat{x}, \hat{x}\rangle^{-1}\right)=0$,

(3) $\lim _{r \rightarrow \infty} r^{2-\beta} \sigma(r)^{-1} \partial_{k} \partial_{l}(\langle A \hat{x}, \hat{x}\rangle)=0$ and

$$
\lim _{r \rightarrow \infty} r^{2-\beta} \sigma(r)^{-1} \partial_{k} \partial_{l}\left(\langle A \hat{x}, \hat{x}\rangle^{-1}\right)=0 \text {, }
$$

(4) $\lim _{r \rightarrow \infty} r \sigma(r)^{-1}\left\{\operatorname{div}(A \hat{x})-(n-1) r^{-1}\right\}=0$ and $\operatorname{div}(A \hat{x})=O\left(r^{-1}\right)$ as $r \rightarrow \infty$,

(5) $\quad \lim _{r \rightarrow \infty} r^{2-\beta} \sigma(r)^{-1} \nabla\{\operatorname{div}(A \hat{x})\}=0$,

(6) $\quad h_{i}(x)=O(1)$ as $r \rightarrow \infty$ for $i=1,2$,

(7) $\lim _{r \rightarrow \infty} r^{1-\beta} \sigma(r)^{-1} \nabla h_{i}(x)=0$ for $i=1,2$.

Proof. We have by direct calculations

$$
\begin{aligned}
& \nabla(\langle A \hat{x}, \hat{x}\rangle)=\langle(\nabla A) \hat{x}, \hat{x}\rangle+2 r^{-1}\{(A-E) \hat{x}-\hat{x}\langle(A-E) \hat{x}, \hat{x}\rangle\}, \\
& \nabla\left(\langle A \hat{x}, \hat{x}\rangle^{-1}\right)=-\langle A \hat{x}, \hat{x}\rangle^{-2} \nabla(\langle A \hat{x}, \hat{x}\rangle) \text {, } \\
& \partial_{k} \partial_{l}(\langle A \hat{x}, \hat{x}\rangle)=\sum_{i, j=1}^{n}\left\{\left(\partial_{k} \partial_{l} a_{i j}\right) \hat{x}_{i} \hat{x}_{j}+\left(\partial_{k} a_{i j}\right) \partial_{l}\left(\hat{x}_{i} \hat{x}_{j}\right)\right. \\
& \left.+\left(\partial_{l} a_{i j}\right) \partial_{k}\left(\hat{x}_{i} \hat{x}_{j}\right)+a_{i j} \partial_{k} \partial_{l}\left(\hat{x}_{i} \hat{x}_{j}\right)\right\}, \\
& \partial_{k} \partial_{l}\left(\langle A \hat{x}, \hat{x}\rangle^{-1}\right)=2\langle A \hat{x}, \hat{x}\rangle^{-3}\left\{\partial_{k}(\langle A \hat{x}, \hat{x}\rangle)\right\}\left\{\partial_{l}(\langle A \hat{x}, \hat{x}\rangle)\right\} \\
& -\langle A \hat{x}, \hat{x}\rangle^{-2} \partial_{k} \partial_{l}(\langle A \hat{x}, \hat{x}\rangle), \\
& \operatorname{div}(A \hat{x})=(n-1) r^{-1}+\operatorname{div}\{(A-E) \hat{x}\},
\end{aligned}
$$




$$
\begin{aligned}
& \partial_{k}\{\operatorname{div}(A \hat{x})\}=\sum_{i, j=1}^{n}\left\{\left(\partial_{k} \partial_{i} a_{i j}\right) \hat{x}_{j}+\left(\partial_{k} a_{i j}\right)\left(\partial_{i} \hat{x}_{j}\right)+\left(\partial_{i} a_{i j}\right)\left(\partial_{k} \hat{x}_{j}\right)\right. \\
&\left.+a_{i j}\left(\partial_{k} \partial_{i} \hat{x}_{j}\right)\right\}, \\
& h_{i}(x)=n-1+2^{-1}\left\{\sigma(r)-\eta(r)-\gamma_{i}\right\}+r\langle A \hat{x}, \hat{x}\rangle^{-1}\left\{\operatorname{div}(A \hat{x})-(n-1) r^{-1}\right\} \\
&+(n-1)\left(\langle A \hat{x}, \hat{x}\rangle^{-1}-1\right)+r\left\langle A \nabla\left(\langle A \hat{x}, \hat{x}\rangle^{-1}\right), \hat{x}\right\rangle, \\
& \partial_{j} h_{i}(x)= \hat{x}_{j}\left\{2^{-1}\left(\sigma^{\prime}(r)-\eta^{\prime}(r)\right)+\langle A \hat{x}, \hat{x}\rangle^{-1} \operatorname{div}(A \hat{x})\right. \\
&\left.+\left\langle A \nabla\left(\langle A \hat{x}, \hat{x}\rangle^{-1}\right), \hat{x}\right\rangle\right\} \\
&+r\left[\partial_{j}\left(\langle A \hat{x}, \hat{x}\rangle^{-1}\right) \operatorname{div}(A \hat{x})+\langle A \hat{x}, \hat{x}\rangle^{-1} \partial_{j}\{\operatorname{div}(A \hat{x})\}\right] \\
&+\sum_{k, l=1}^{n} r\left\{\hat{x_{k}}\left(\partial_{j} a_{k l}\right) \partial_{l}\left(\langle A \hat{x}, \hat{x}\rangle^{-1}\right)+\hat{x}_{k} a_{k l} \partial_{j} \partial_{l}\left(\langle A \hat{x}, \hat{x}\rangle^{-1}\right)\right. \\
&\left.+a_{k l} \partial_{l}\left(\langle A \hat{x}, \hat{x}\rangle^{-1}\right) \partial_{j} \hat{x}_{k}\right\},
\end{aligned}
$$

where $E=\left(\delta_{i j}\right)$ is the $n \times n$ identity matrix. So noting $(\mathrm{F})$ and

$$
\begin{aligned}
& \partial_{k} \hat{x}_{i}=O\left(r^{-1}\right) \text { and } \partial_{k} \partial_{l} \hat{x}_{i}=O\left(r^{-2}\right) \quad \text { as } r \rightarrow \infty, \\
& \lim _{r \rightarrow \infty} r^{\beta} \sigma(r)=+\infty \quad(\text { by }(\mathrm{F} 3)),
\end{aligned}
$$

we have the assertions.

Lemma 2.7. There exist some constants $C_{4}>0$ and $R_{1} \geq R_{0}$ such that for any $r \geq R_{1}$ and any $w \in H_{\text {loc }}^{2}(\Omega)$ we have

$$
G_{1}(x ; w) \geq C_{4} \sigma(r)\left\{|\langle A D w, \hat{x}\rangle|^{2}+r^{2 \beta-2}|w|^{2}\right\} .
$$

Proof. In the sequel $\varepsilon_{i}(r)(i=1,2, \ldots)$ means a positive function for $r>R_{0}$ which tends to 0 as $r \rightarrow \infty$. Choose a constant $a_{1}^{\prime}$ satisfying $1<a_{1}^{\prime}$ $<a_{1}$. Using

$$
\begin{gathered}
|\langle A(x) \xi, \eta\rangle| \leq\langle A(x) \xi, \bar{\xi}\rangle^{1 / 2}\langle A(x) \eta, \bar{\eta}\rangle^{1 / 2} \\
\text { for any } x \in \Omega \text { and any } \xi, \eta \in \mathbb{C}^{n}, \\
\left\langle A\left\{D w-\hat{x}\langle A \hat{x}, \hat{x}\rangle^{-1}\langle A D w, \hat{x}\rangle\right\},\left\{\overline{D w}-\hat{x}\langle A \hat{x}, \hat{x}\rangle^{-1}\langle A \overline{D w}, \hat{x}\rangle\right\}\right\rangle \\
=\langle A D w, \overline{D w}\rangle-\langle A \hat{x}, \hat{x}\rangle^{-1}|\langle A D w, \hat{x}\rangle|^{2}
\end{gathered}
$$

for any $x \in \Omega$ and any $w \in H_{l o c}^{2}(\Omega)$, and noting Lemma 2.6, conditions (A 3), (F 7) and $(\mathrm{F} 8)$, we have the following:

$$
\begin{aligned}
& 2 r \operatorname{Re}\left[\left\langle A D w, \nabla\left(\langle A \hat{x}, \hat{x}\rangle^{-1}\right)\right\rangle\langle A \overline{D w}, \hat{x}\rangle\right] \geq-\varepsilon_{1}(r) \sigma(r)\langle A D w, \overline{D w}\rangle, \\
& 2\left\{\langle A \hat{x}, \hat{x}\rangle^{-1}|A D w|^{2}-\langle A D w, \overline{D w}\rangle\right\}=2\langle A \hat{x}, \hat{x}\rangle^{-1}\langle A D w,(A-E) \overline{D w}\rangle \\
& \quad+2\left(\langle A \hat{x}, \hat{x}\rangle^{-1}-1\right)\langle A D w, \overline{D w}\rangle \geq-\varepsilon_{2}(r) \sigma(r)\langle A D w, \overline{D w}\rangle,
\end{aligned}
$$


$2 r\langle A \hat{x}, \hat{x}\rangle^{-1} \operatorname{Re}[\langle(\langle A D w, \nabla\rangle A) \overline{D w}, \hat{x}\rangle] \geq-\varepsilon_{3}(r) \sigma(r)\langle A D w, \overline{D w}\rangle$, $-r\langle A \hat{x}, \hat{x}\rangle^{-1} \operatorname{Re}[\langle(\langle\hat{x}, A \nabla\rangle A) D w, \overline{D w}\rangle] \geq-\varepsilon_{4}(r) \sigma(r)\langle A D w, \overline{D w}\rangle$, $-2\langle A \hat{x}, \hat{x}\rangle^{-1} \operatorname{Re}\left[\sqrt{-1}\left\langle A B A x,\left(D w-\hat{x}\langle A \hat{x}, \hat{x}\rangle^{-1}\langle A D w, \hat{x}\rangle\right)\right\rangle \bar{w}\right]$

$$
\begin{aligned}
& \geq-2\langle A \hat{x}, \hat{x}\rangle^{-1}\{\langle A B A x, B A x\rangle\}^{1 / 2}|w| \\
& \cdot\left\{\langle A D w, \overline{D w}\rangle-\langle A \hat{x}, \hat{x}\rangle^{-1}|\langle A D w, \hat{x}\rangle|^{2}\right\}^{1 / 2} \\
& \geq-\left(2-\gamma_{1}-\eta\right) a_{2}^{-1}\left\{\langle A D w, \overline{D w}\rangle-\langle A \hat{x}, \hat{x}\rangle^{-1}|\langle A D w, \hat{x}\rangle|^{2}\right\} \\
&-\left(2-\gamma_{1}-\eta\right)^{-1} a_{2}\langle A \hat{x}, \hat{x}\rangle^{-2}\langle A B A x, B A x\rangle|w|^{2},
\end{aligned}
$$

$2 r\langle A \hat{x}, \hat{x}\rangle^{-1} \operatorname{Re}\left[\langle A D w, \hat{x}\rangle \overline{q_{2} w}\right]$

$$
\begin{aligned}
\geq-a_{1}^{\prime-1} \sigma(r)\langle A \hat{x}, \hat{x}\rangle^{-1}|\langle A D w, \hat{x}\rangle|^{2} \\
\quad-a_{1}^{\prime} \sigma(r)^{-1}\langle A \hat{x}, \hat{x}\rangle^{-1}\left|r q_{2}\right|^{2}|w|^{2},
\end{aligned}
$$

$\operatorname{Re}\left[\left\langle A D w, \nabla h_{1}\right\rangle \bar{w}\right] \geq-\varepsilon_{5}(r) \sigma(r)\left\{\langle A D w, \overline{D w}\rangle+r^{2 \beta-2}|w|^{2}\right\}$,

$(2 r)^{-1} h_{1}\left\{\eta(r)+\sigma(r)+\gamma_{1}-2\right\} \operatorname{Re}[\langle A D w, \hat{x}\rangle \bar{w}]$

$$
\begin{aligned}
\geq-2^{-1} & \left(1-a_{1}^{\prime-1}\right) \sigma(r)\langle A \hat{x}, \hat{x}\rangle^{-1}|\langle A D w, \hat{x}\rangle|^{2} \\
& -8^{-1} h_{1}^{2}\left(1-a_{1}^{\prime-1}\right)^{-1}\langle A \hat{x}, \hat{x}\rangle r^{-2} \sigma(r)^{-1}\left(\eta+\sigma+\gamma_{1}-2\right)^{2}|w|^{2},
\end{aligned}
$$

$h_{1} \operatorname{Re}\left[q_{2}\right] \geq-\left(a_{1}-a_{1}^{\prime}\right)\langle A \hat{x}, \hat{x}\rangle^{-1} \sigma(r)^{-1}\left|r q_{2}\right|^{2}$

$$
-4^{-1}\left(a_{1}-a_{1}^{\prime}\right)^{-1} \sigma(r) h_{1}^{2}\langle A \hat{x}, \hat{x}\rangle r^{-2} \text {. }
$$

Noting Definition 2.4 and

$$
\begin{aligned}
\langle A D w, \overline{D w}\rangle= & \left\{\langle A D w, \overline{D w}\rangle-\langle A \hat{x}, \hat{x}\rangle^{-1}|\langle A D w, \hat{x}\rangle|^{2}\right\} \\
& +\langle A \hat{x}, \hat{x}\rangle^{-1}|\langle A D w, \hat{x}\rangle|^{2},
\end{aligned}
$$

we have

$$
\begin{gathered}
G_{1}(x ; w) \geq \sigma(r)\left\{2^{-1}\left(1-a_{1}^{\prime-1}\right)-\sum_{i=1}^{5} \varepsilon_{i}(r)\right\}\langle A \hat{x}, \hat{x}\rangle^{-1}|\langle A D w, \hat{x}\rangle|^{2} \\
+\left\{\left(2-\gamma_{1}-\eta(r)\right)\left(1-a_{2}^{-1}\right)-\sigma(r) \sum_{i=1}^{5} \varepsilon_{i}(r)\right\} \\
\cdot\left\{\langle A D w, \overline{D w}\rangle-\langle A \hat{x}, \hat{x}\rangle^{-1}|\langle A D w, \hat{x}\rangle|^{2}\right\} \\
-\left\{r\langle A \hat{x}, \hat{x}\rangle^{-1}\left\langle A \nabla q_{1}, \hat{x}\right\rangle+\left(\gamma_{1}+\eta(r)\right) q_{1}+a_{1} \sigma(r)^{-1}\right. \\
\cdot\langle A \hat{x}, \hat{x}\rangle^{-1}\left|r q_{2}\right|^{2} \\
\left.+a_{2}\left(2-\gamma_{1}-\eta(r)\right)^{-1}\langle A \hat{x}, \hat{x}\rangle^{-2}\langle A B A x, B A x\rangle\right\}|w|^{2} \\
-\left\{\varepsilon_{5}(r) \sigma(r) r^{2 \beta-2}+8^{-1} h_{1}^{2}\left(1-a_{1}^{\prime-1}\right)^{-1}\right. \\
\cdot\langle A \hat{x}, \hat{x}\rangle\left(\eta+\sigma+\gamma_{1}-2\right)^{2} \sigma(r)^{-1} r^{-2}
\end{gathered}
$$




$$
+4^{-1}\left(a_{1}-a_{1}^{\prime}\right)^{-1} h_{1}^{2}\left\langle A \hat{x}, \hat{x}>\sigma(r) r^{-2}\right\}|w|^{2} .
$$

We note that we have by (F1) and (F3)

$$
\lim _{r \rightarrow \infty} r^{\beta} \sigma(r)=+\infty
$$

Therefore by (A3), (F1), (F2), (F5) and Lemma 2.6 there exist some constants $C_{4}>0$ and $R_{1} \geq R_{0}$ such that for any $r \geq R_{1}$ we have

$$
\begin{aligned}
& \left\{2^{-1}\left(1-a_{1}^{\prime-1}\right)-\sum_{i=1}^{5} \varepsilon_{i}(r)\right\}\langle A \hat{x}, \hat{x}\rangle^{-1} \geq C_{4}, \\
& \left(2-\gamma_{1}-\eta(r)\right)\left(1-a_{2}^{-1}\right)-\sigma(r) \sum_{i=1}^{5} \varepsilon_{i}(r) \geq 0 \\
& -\left\{r\langle A \hat{x}, \hat{x}\rangle^{-1}\left\langle A D q_{1}, \hat{x}\right\rangle+\left(\gamma_{1}+\eta(r)\right) q_{1}+a_{1} \sigma(r)^{-1}\langle A \hat{x}, \hat{x}\rangle^{-1}\left|r q_{2}\right|^{2}\right. \\
& \left.\quad+a_{2}\left(2-\gamma_{1}-\eta(r)\right)^{-1}\langle A \hat{x}, \hat{x}\rangle^{-2}\langle A B A x, B A x\rangle\right\} \\
& \geq 2 C_{4} r^{2 \beta-2} \sigma(r), \\
& -\left\{\varepsilon_{5}(r) r^{2 \beta-2} \sigma(r)+8^{-1} h_{1}^{2}\left(1-a_{1}^{\prime-1}\right)^{-1}\langle A \hat{x}, \hat{x}\rangle\left(\eta+\sigma+\gamma_{1}-2\right)^{2} r^{-2} \sigma(r)^{-1}\right. \\
& \left.\quad+4^{-1}\left(a_{1}-a_{1}^{\prime}\right)^{-1} h_{1}^{2}\langle A \hat{x}, \hat{x}\rangle r^{-2} \sigma(r)\right\} \\
& \geq-C_{4} r^{2 \beta-2} \sigma(r) .
\end{aligned}
$$

So using

$$
\langle A D w, \overline{D w}\rangle-\langle A \hat{x}, \hat{x}\rangle^{-1}|\langle A D w, \hat{x}\rangle|^{2} \geq 0,
$$

we have the assertion.

Lemma 2.8. There exist some constants $C_{5}>0, C_{6}>0$ and $R_{2} \geq R_{0}$ such that for any $r \geq R_{2}$ and $w \in H_{\text {loc }}^{2}(\Omega)$ we have

$$
G_{2}(x ; w) \geq C_{5} r^{2 \beta-2} \sigma(r)|w|^{2}-C_{6}|\langle A D w, \hat{x}\rangle|^{2} .
$$

Proof. We use the same estimates as given in the proof of Lemma 2.7 except for the following: Choose a constant $a_{3}^{\prime}$ to satisfy $0<a_{3}^{\prime}<a_{3}$, and we have

$$
\begin{aligned}
&- 2\langle A \hat{x}, \hat{x}\rangle^{-1} \operatorname{Re}\left[\sqrt{-1}\left\langle A B A x,\left(D w-\hat{x}\langle A \hat{x}, \hat{x}\rangle^{-1}\langle A D w, \hat{x}\rangle\right)\right\rangle \bar{w}\right] \\
& \geq-a_{4}^{-1}\left(2-\gamma_{2}-\eta(r)\right)\left\{\langle A D w, \overline{D w}\rangle-\langle A \hat{x}, \hat{x}\rangle^{-1}|\langle A D w, \hat{x}\rangle|^{2}\right\} \\
&-a_{4}\left(2-\gamma_{2}-\eta(r)\right)^{-1}\langle A \hat{x}, \hat{x}\rangle^{-2}\langle A B A x, B A x\rangle|w|^{2}, \\
& 2 r\langle A \hat{x}, \hat{x}\rangle^{-1} \operatorname{Re}\left[\langle A D w, \hat{x}\rangle \overline{q_{2} w}\right] \\
& \geq-a_{3}^{\prime-1} \sigma(r)\langle A \hat{x}, \hat{x}\rangle^{-1}|\langle A D w, \hat{x}\rangle|^{2}-a_{3}^{\prime} \sigma(r)^{-1}\langle A \hat{x}, \hat{x}\rangle^{-1}\left|r q_{2}\right|^{2}|w|^{2}, \\
&(2 r)^{-1} h_{2}\left(\eta+\sigma+\gamma_{2}-2\right) \operatorname{Re}[\langle A D w, \hat{x}\rangle \bar{w}]
\end{aligned}
$$




$$
\begin{aligned}
\geq & -2^{-1} \sigma(r)\langle A \hat{x}, \hat{x}\rangle^{-1}|\langle A D w, \hat{x}\rangle|^{2} \\
& -8^{-1} h_{2}^{2}\langle A \hat{x}, \hat{x}\rangle\left(\eta+\sigma+\gamma_{2}-2\right)^{2} r^{-2} \sigma(r)^{-1}|w|^{2}, \\
h_{2} \operatorname{Re} & {\left[q_{2}\right] \geq-\left(a_{3}-a_{3}^{\prime}\right)\langle A \hat{x}, \hat{x}\rangle^{-1} \sigma(r)^{-1}\left|r q_{2}\right|^{2} } \\
& -4^{-1}\left(a_{3}-a_{3}^{\prime}\right)^{-1}\langle A \hat{x}, \hat{x}\rangle h_{2}^{2} r^{-2} \sigma(r) .
\end{aligned}
$$

Combine the above estimates with the remaining ones given in the proof of Lemma 2.7, and we have by Definition 2.4

$$
\begin{gathered}
G_{2}(x ; w) \geq \sigma(r)\left\{2^{-1}-\sum_{i=1}^{5} \varepsilon_{i}(r)-a_{3}^{\prime-1}\right\}\langle A \hat{x}, \hat{x}\rangle^{-1}|\langle A D w, \hat{x}\rangle|^{2} \\
+\left\{\left(2-\gamma_{2}-\eta\right)\left(1-a_{4}^{-1}\right)-\sigma(r) \sum_{i=1}^{5} \varepsilon_{i}(r)\right\}\{\langle A D w, \overline{D w}\rangle \\
\left.-\langle A \hat{x}, \hat{x}\rangle^{-1}|\langle A D w, \hat{x}\rangle|^{2}\right\} \\
-\left\{r\langle A \hat{x}, \hat{x}\rangle^{-1}\left\langle A D q_{1}, \hat{x}\right\rangle+\left(\gamma_{2}+\eta(r)\right) q_{1}+a_{3} \sigma(r)^{-1}\langle A \hat{x}, \hat{x}\rangle^{-1}\left|r q_{2}\right|^{2}\right. \\
\left.+a_{4}\left(2-\gamma_{2}-\eta(r)\right)^{-1}\langle A \hat{x}, \hat{x}\rangle^{-2}\langle A B A x, B A x\rangle\right\}|w|^{2} \\
-\left\{\varepsilon_{5}(r) r^{2 \beta-2} \sigma(r)+8^{-1} h_{2}^{2}\langle A \hat{x}, \hat{x}\rangle\left(\eta+\sigma+\gamma_{2}-2\right)^{2} r^{-2} \sigma(r)^{-1}\right. \\
\left.+4^{-1}\left(a_{3}-a_{3}^{\prime}\right)^{-1}\langle A \hat{x}, \hat{x}\rangle h_{2}^{2} r^{-2} \sigma(r)\right\}|w|^{2} .
\end{gathered}
$$

By (F1), (F2), (F6), Lemma 2.6 and $\lim _{r \rightarrow \infty} r^{\beta} \sigma(r)=+\infty$, there exist some constants $C_{5}>0, C_{6}>0$ and $R_{2} \geq R_{0}$ such that for any $r \geq R_{2}$ we have

$$
\begin{aligned}
& \sigma(r)\left\{2^{-1}-\sum_{i=1}^{5} \varepsilon_{i}(r)-a_{3}^{\prime-1}\right\}\langle A \hat{x}, \hat{x}\rangle^{-1} \geq-C_{6}, \\
& \left(2-\gamma_{2}-\eta(r)\right)\left(1-a_{4}^{-1}\right)-\sigma(r) \sum_{i=1}^{5} \varepsilon_{i}(r) \geq 0, \\
& -\left\{r\langle A \hat{x}, \hat{x}\rangle^{-1}\left\langle A \nabla q_{1}, \hat{x}\right\rangle+\left(\gamma_{2}+\eta(r)\right) q_{1}+a_{3}\langle A \hat{x}, \hat{x}\rangle^{-1} \sigma(r)^{-1}\left|r q_{2}\right|^{2}\right. \\
& \left.\quad+a_{4}\left(2-\gamma_{2}-\eta(r)\right)^{-1}\langle A \hat{x}, \hat{x}\rangle^{-2}\langle A B A x, B A x\rangle\right\} \\
& \geq 2 C_{5} r^{2 \beta-2} \sigma(r), \\
& -\left\{\varepsilon_{5}(r) r^{2 \beta-2} \sigma(r)+8^{-1} h_{2}^{2}\langle A \hat{x}, \hat{x}\rangle\left(\eta+\sigma+\gamma_{2}-2\right)^{2} r^{-2} \sigma(r)^{-1}\right. \\
& \left.\quad+4^{-1}\left(a_{3}-a_{3}^{\prime}\right)^{-1}\langle A \hat{x}, \hat{x}\rangle h_{2}^{2} r^{-2} \sigma(r)\right\} \\
& \geq-C_{5} r^{2 \beta-2} \sigma(r) .
\end{aligned}
$$

So we have the assertion.

Lemma 2.9. There exists some constant $R_{3} \geq R_{2}$ such that for any constant $m \geq 1$ and any $t, s$ satisfying $t \geq s \geq R_{3}$ we have

$$
F\left(t ; m r^{\alpha}, f_{2}, g_{2}\right) \geq F\left(s ; m r^{\alpha}, f_{2}, g_{2}\right) \text {. }
$$

Proof. In Lemma 2.5 let $i=2$ and $\rho(r)=m r^{\alpha}$. Then we have 


$$
\begin{aligned}
& 4 r \rho^{\prime}\langle A \hat{x}, \hat{x}\rangle^{-1}|\langle A D v, \hat{x}\rangle|^{2}=4 m \alpha r^{\alpha}\langle A \hat{x}, \hat{x}\rangle^{-1}|\langle A D v, \hat{x}\rangle|^{2}, \\
& 2\left\{r \rho^{\prime \prime}+r \rho^{\prime}\langle A \hat{x}, \hat{x}\rangle^{-1} \operatorname{div}(A \hat{x})+h_{2} \rho^{\prime}\right\} \operatorname{Re}[\langle A D v, \hat{x}\rangle \bar{v}] \\
& =2 m \alpha r^{\alpha-1}\left\{\alpha-1+r\langle A \hat{x}, \hat{x}\rangle^{-1} \operatorname{div}(A \hat{x})+h_{2}\right\} \operatorname{Re}[\langle A D v, \hat{x}\rangle \bar{v}] \\
& \geq-m \alpha r^{\alpha}\langle A \hat{x}, \hat{x}\rangle^{-1}|\langle A D v, \hat{x}\rangle|^{2} \\
& \quad-m \alpha r^{\alpha-2}\left\{\alpha-1+r\langle A \hat{x}, \hat{x}\rangle^{-1} \operatorname{div}(A \hat{x})+h_{2}\right\}^{2}\langle A \hat{x}, \hat{x}\rangle|v|^{2}, \\
& \left(\gamma_{2}+\eta(r)\right) \rho^{\prime 2}+2 r \rho^{\prime} \rho^{\prime \prime}-r \rho^{\prime 2}\left\langle A D\left(\langle A \hat{x}, \hat{x}\rangle^{-1}\right), \hat{x}\right\rangle \\
& \quad+h_{2} \rho^{\prime \prime}+h_{2} \rho^{\prime}\langle A x, x\rangle^{-1} \operatorname{div}(A \hat{x}) \\
& =m^{2} \alpha^{2} r^{2 \alpha-2}\left\{\gamma_{2}+\eta(r)+2 \alpha-2-r\left\langle A \nabla\left(\langle A \hat{x}, \hat{x}\rangle{ }^{-1}\right), \hat{x}\right\rangle\right\} \\
& +h_{2} m \alpha r^{\alpha-2}\left\{\alpha-1+r\langle A \hat{x}, \hat{x}\rangle^{-1} \operatorname{div}(A \hat{x})\right\} .
\end{aligned}
$$

By Lemma 2.8 we have for any $r \geq R_{2}$

$$
\begin{aligned}
& G_{2}(x ; v)+4 r \rho^{\prime}\langle A \hat{x}, \hat{x}\rangle^{-1}|\langle A D v, \hat{x}\rangle|^{2} \\
& +2\left\{r \rho^{\prime \prime}+r \rho^{\prime}\langle A \hat{x}, \hat{x}\rangle^{-1} \operatorname{div}(\mathrm{A} \hat{x})+h_{2} \rho^{\prime}\right\} \operatorname{Re}[\langle A D v, \hat{x}\rangle \bar{v}] \\
& +\langle A \hat{x}, \hat{x}\rangle\left\{\left(\gamma_{2}+\eta(r)\right) \rho^{\prime 2}+2 r \rho^{\prime} \rho^{\prime \prime}-r \rho^{\prime 2}\left\langle A D\left(\langle A \hat{x}, \hat{x}\rangle{ }^{-1}\right), \hat{x}\right\rangle\right. \\
& \left.\quad+h_{2} \rho^{\prime \prime}+h_{2} \rho^{\prime}\langle A \hat{x}, \hat{x}\rangle^{-1} \operatorname{div}(A \hat{x})\right\}|v|^{2} \\
& \quad C_{5} r^{2 \beta-2} \sigma(r)|v|^{2}-C_{6}|\langle A D v, \hat{x}\rangle|^{2}+3 m \alpha r^{\alpha}\langle A \hat{x}, \hat{x}\rangle^{-1}|\langle A D v, \hat{x}\rangle|^{2} \\
& +m \alpha r^{\alpha-2}\left[\left\{\gamma_{2}+\eta(r)+2 \alpha-2-r\left\langle A \nabla\left(\langle A \hat{x}, \hat{x}\rangle^{-1}\right), \hat{x}\right\rangle\right\} m \alpha r^{\alpha}\right. \\
& +h_{2}\left\{\alpha-1+r\langle A \hat{x}, \hat{x}\rangle^{-1} \operatorname{div}(A \hat{x})\right\} \\
& \left.-\left\{\alpha-1+r\langle A \hat{x}, \hat{x}\rangle^{-1} \operatorname{div}(A \hat{x})+h_{2}\right\}^{2}\langle A \hat{x}, \hat{x}\rangle\right]|v|^{2} .
\end{aligned}
$$

Noting Lemma 2.6 and (F2), there exists some constant $R_{3} \geq R_{2}$ such that for any $r \geq R_{3}$ and any constant $m \geq 1$ we have

$$
\begin{aligned}
& 3 m \alpha r^{\alpha}\langle A \hat{x}, \hat{x}\rangle^{-1}-C_{6} \geq 0, \\
& \left\{\gamma_{2}+\eta(r)+2 \alpha-2-r\left\langle A D\left(\langle A \hat{x}, \hat{x}\rangle^{-1}\right), \hat{x}\right\rangle\right\} m \alpha r^{\alpha} \\
& +h_{2}\left\{\alpha-1+r\langle A \hat{x}, \hat{x}\rangle^{-1} \operatorname{div}(A \hat{x})\right\}-\left\{\alpha-1+r\langle A \hat{x}, \hat{x}\rangle^{-1} \operatorname{div}(A \hat{x})\right. \\
& \left.+h_{2}\right\}^{2}\langle A \hat{x}, \hat{x}\rangle
\end{aligned}
$$

$\geq 0$.

So we have the assertion.

We intend to prove Theorem 1.1(1) by reduction to a contradiction.

Lemma 2.10. If

$$
\liminf _{R \rightarrow \infty} R^{\left(\gamma_{1} / 2\right)} \Phi(R) \int_{|x|=R}\left[|\langle A D u, \hat{x}\rangle|^{2}+\left\{r^{-2}+\left(q_{1}\right)_{-}\right\}|u|^{2}\right] d S=0,
$$


then we have for any $m=0,1,2, \ldots$

$$
\int_{|x|>R_{0}} r^{m \alpha+\left(\gamma_{1} / 2\right)-1} \Phi(r) \sigma(r)\left\{|\langle A D u, \hat{x}\rangle|^{2}+r^{2 \beta-2}|u|^{2}\right\} d x<\infty .
$$

Proof. By Lemma 2.2 and Lemma 2.6, there exist some constants $C_{7}>0$ and $R_{4} \geq R_{0}$ such that for any $t \geq R_{4}$ we have

$$
\begin{aligned}
F( & \left.; 0, f_{1}, g_{1}\right) \\
= & t^{\left(\gamma_{1} / 2\right)} \Phi(t) \int_{|x|=t}\left[2\langle A \hat{x}, \hat{x}\rangle^{-1}|\langle A D u, \hat{x}\rangle|^{2}-\langle A D u, \overline{D u}\rangle\right. \\
& \left.+h_{1} r^{-1} \operatorname{Re}[\langle A D u, \hat{x}\rangle \bar{u}]-q_{1}|u|^{2}\right] d S \\
\leq & C_{7} t^{\left(\gamma_{1} / 2\right)} \Phi(t) \int_{|x|=t}\left[|\langle A D u, \hat{x}\rangle|^{2}+\left\{r^{-2}+\left(q_{1}\right)_{-}\right\}|u|^{2}\right] d S .
\end{aligned}
$$

So we have

$$
\liminf _{t \rightarrow \infty} F\left(t ; 0, f_{1}, g_{1}\right) \leq 0
$$

Letting $t \rightarrow \infty$ along the suitable subsequence in Lemma 2.5, we have, by Lemma 2.7, for any $s \geq R_{1}$

$$
C_{4} \int_{|x|>s} r^{\left(\gamma_{1} / 2\right)-1} \Phi(r) \sigma(r)\left\{|\langle A D u, \hat{x}\rangle|^{2}+r^{2 \beta-2}|u|^{2}\right\} d x \leq-F\left(s ; 0, f_{1}, g_{1}\right),
$$

which shows that the assertion holds for $m=0$. By the above estimate, and by Definitions 2.1 and 2.4 and Lemma 2.3 we have

$$
\begin{aligned}
& C_{4} \int_{R_{1}}^{t} s^{(m+1) \alpha-1} d s \int_{|x|>s} r^{\left(\gamma_{1} / 2\right)-1} \Phi(r) \sigma(r)\left\{|\langle A D u, \hat{x}\rangle|^{2}+r^{2 \beta-2}|u|^{2}\right\} d x \\
& \leq-\int_{R_{1}}^{t} s^{(m+1) \alpha-1} F\left(s ; 0, f_{1}, g_{1}\right) d s \\
& =\int_{R_{1}<|x|<t} r^{(m+1) \alpha+\left(\gamma_{1} / 2\right)-1} \Phi(r)\left\{\langle A D u, \overline{D u}\rangle+q_{1}|u|^{2}\right. \\
& =F\left(t ; 0,0, r^{(m+1) \alpha+\left(\gamma_{1} / 2\right)-1} \Phi(r)\right)-F\left(R_{1} ; 0,0, r^{(m+1) \alpha+\left(\gamma_{1} / 2\right)-1} \Phi(r)\right) \\
& \quad-\int_{R_{1}<|x|<t}^{r^{(m+1) \alpha+\left(\gamma_{1} / 2\right)-1} \Phi(r)[2\langle A \hat{x}, \hat{x}\rangle)^{-1}|\langle A D u, \hat{x}\rangle|^{2}} \\
& \quad+\left\{(m+1) \alpha+2^{-1}\left(\eta(r)+\sigma(r)+\gamma_{1}\right)-1+h_{1}\right\} r^{-1} \operatorname{Re}[\langle A D u, \hat{x}\rangle \bar{u}] \\
& \left.\quad+\operatorname{Re}\left[q_{2}\right]|u|^{2}\right] d x .
\end{aligned}
$$


Now we assume that the statement is true for $m$. Then by (G2), (F3), $\alpha<\beta$ and Lemma 2.6 we have for any $t \geq R_{1}$

$$
\begin{aligned}
& -\int_{R_{1}<|x|<t} r^{(m+1) \alpha+\left(\gamma_{1} / 2\right)-1} \Phi(r)\left[2\langle A \hat{x}, \hat{x}\rangle^{-1}|\langle A D u, \hat{x}\rangle|^{2}\right. \\
& \quad+r^{-1}\left\{(m+1) \alpha+2^{-1}\left(\eta(r)+\sigma(r)+\gamma_{1}\right)-1+h_{1}\right\} \operatorname{Re}[\langle A D u, \hat{x}\rangle \bar{u}] \\
& \left.\quad+\operatorname{Re}\left[q_{2}\right]|u|^{2}\right] d x \\
& \leq \int_{|x|>R_{1}} r^{m \alpha+\left(\gamma_{1} / 2\right)+2 \beta-3} \Phi(r) \sigma(r)\left[8^{-1} r^{\alpha-2 \beta} \sigma(r)^{-1}\{(m+1) \alpha\right. \\
& \left.\left.+2^{-1}\left(\eta+\sigma+\gamma_{1}\right)-1+h_{1}\right\}^{2}\langle A \hat{x}, \hat{x}\rangle+C_{3} r^{\alpha-\beta}\right]|u|^{2} d x \\
& <+\infty
\end{aligned}
$$

On the other hand by Lemma 2.2 we have

$$
\begin{aligned}
& F\left(t ; 0,0, r^{(m+1) \alpha+\left(\gamma_{1} / 2\right)-1} \Phi(r)\right) \\
& \quad=\int_{|x|=t} r^{(m+1) \alpha+\left(\gamma_{1} / 2\right)-1} \Phi(r) \operatorname{Re}[\langle A D u, \hat{x}\rangle \bar{u}] d S \\
& \quad \leq 2^{-1} \int_{|x|=t} r^{m \alpha+\left(\gamma_{1} / 2\right)} \Phi(r) \sigma(r)\left[|\langle A D u, \hat{x}\rangle|^{2}+r^{2 \beta-2}\left\{r^{\beta-\alpha} \sigma(r)\right\}^{-2}|u|^{2}\right] d S .
\end{aligned}
$$

Noting that the assertion holds for $m$, we have by (F3)

$$
\liminf _{t \rightarrow \infty} F\left(t ; 0,0, r^{(m+1) \alpha+\left(\gamma_{1} / 2\right)-1} \Phi(r)\right) \leq 0
$$

Therefore we have

$$
\begin{gathered}
+\infty>\int_{R_{1}}^{\infty} s^{(m+1) \alpha-1} d s \int_{|x|>s} r^{\left(\gamma_{1} / 2\right)-1} \Phi(r) \sigma(r)\left\{|\langle A D u, \hat{x}\rangle|^{2}+r^{2 \beta-2}|u|^{2}\right\} d x \\
=(m+1)^{-1} \alpha^{-1} \int_{|x|>R_{1}}\left\{r^{(m+1) \alpha}-R_{1}^{(m+1) \alpha}\right\} r^{\left(\gamma_{1} / 2\right)-1} \Phi(r) \sigma(r) \\
\times\left\{|\langle A D u, \hat{x}\rangle|^{2}+r^{2 \beta-2}|u|^{2}\right\} d x,
\end{gathered}
$$

which shows that the assertion is true for $m+1$.

Lemma 2.11. There exist some constants $R_{5}>R_{0}$ and $C_{8}>0$ such that for any real-valued function $\psi(r) \in C_{0}^{1}\left(R_{5}, \infty\right)$ we have

$$
\int_{\Omega} \psi(r)^{2}\left\{|D u|^{2}+\left(q_{1}\right)_{-}|u|^{2}\right\} d x \leq C_{8} \int_{\Omega}\left\{r^{\max \left\{\delta_{1}, \delta_{2}\right\}} \psi(r)^{2}+\psi^{\prime}(r)^{2}\right\}|u|^{2} d x .
$$

Proof. See Lemma 2.3 of Uchiyama [3], where (A), (G) and $\lim _{r \rightarrow \infty}\left\{a_{i j}(x)\right.$ 
$\left.-\delta_{i j}\right\}=0$ have been used.

Lemma 2.12. Let $R_{5}$ be the one given in Lemma 2.11. There exists some constant $C_{9}>0$ such that for any $R \geq R_{5}$ and any real-valued function $\zeta(r) \in C^{1}\left[R_{5}, \infty\right)$ satisfying

$$
\begin{aligned}
& \int_{|x|>R_{5}}\left[r^{\max \left\{\delta_{1}, \delta_{2}\right\}} \zeta(r)^{2}+\zeta^{\prime}(r)^{2}\right]|u|^{2} d x<\infty, \\
& \liminf _{t \rightarrow \infty} \int_{t<|x|<t+1} \zeta(r)^{2}|u|^{2} d x=0,
\end{aligned}
$$

we have

$$
\begin{aligned}
& \int_{|x|>R+1} \zeta(r)^{2}\left\{|D u|^{2}+\left(q_{1}\right)_{-}|u|^{2}\right\} d x \\
& \quad \leq C_{9} \int_{|x|>R}\left\{r^{\max \left\{\delta_{1}, \delta_{2}\right\}} \zeta(r)^{2}+\zeta^{\prime}(r)^{2}\right\}|u|^{2} d x+C_{9} \int_{R<|x|<R+1} \zeta(r)^{2}|u|^{2} d x .
\end{aligned}
$$

Proof. See Lemma 2.4 of Uchiyama [3], which can be obtained from Lemma 2.11.

Lemma 2.13. There exist some constants $\delta_{3}>0, C_{10}>0$ and $C_{11}>0$ such that for any $r \geq R_{0}$ we have

$$
C_{10} r^{-\delta_{3}} \leq \Phi(r) \leq C_{11} r^{\delta_{3}} .
$$

Proof. By (F2) there exist some constants $\delta_{3}>0$ and $R_{6} \geq R_{0}$ such that for any $r \geq R_{6}$ we have

$$
-2 \delta_{3} \leq \eta(r)+\sigma(r) \leq 2 \delta_{3} .
$$

Then we have for any $r \geq R_{6}$

$$
\begin{aligned}
\frac{1}{2} \int_{R_{0}}^{R_{6}} \frac{\eta(r)+\sigma(r)}{r} d r-\delta_{3} \log \frac{r}{R_{6}} & \leq \frac{1}{2} \int_{R_{0}}^{r} \frac{\eta(r)+\sigma(r)}{r} d r \\
& \leq \frac{1}{2} \int_{R_{0}}^{R_{6}} \frac{\eta(r)+\sigma(r)}{r} d r+\delta_{3} \log \frac{r}{R_{6}} .
\end{aligned}
$$

Note that $\Phi(r)$ is continuous in $\left[R_{0}, \infty\right)$, where $R_{0} \geq 1$. Then letting

$$
\begin{aligned}
& C_{10}=\min \left\{R_{6}^{\delta_{3}} \exp \left\{\frac{1}{2} \int_{R_{0}}^{R_{6}} \frac{\eta(r)+\sigma(r)}{r} d r\right\}, \min _{R_{0} \leq r \leq R_{6}} r^{\delta_{3}} \Phi(r)\right\}>0, \\
& C_{11}=\max \left\{R_{6}^{-\delta_{3}} \exp \left\{\frac{1}{2} \int_{R_{0}}^{R_{6}} \frac{\eta(r)+\sigma(r)}{r} d r\right\}, \max _{R_{0} \leq r \leq R_{6}} r^{-\delta_{3}} \Phi(r)\right\}>0
\end{aligned}
$$


we have the assertion.

Lemma 2.14. If

$$
\liminf _{R \rightarrow \infty} R^{\left(\gamma_{1} / 2\right)} \Phi(R) \int_{|x|=R}\left[|\langle A D u, \hat{x}\rangle|^{2}+\left\{r^{-2}+\left(q_{1}\right)_{-}\right\}|u|^{2}\right] d S=0,
$$

then we have for any constant $m>0$

$$
\int_{|x|>R_{0}} r^{m}\left[|D u|^{2}+\left\{1+\left(q_{1}\right)_{-}\right\}|u|^{2}\right] d x<\infty .
$$

Proof. By Lemmas 2.10 and 2.13 and (F3) we have for any $m>0$

Let

$$
\int_{|x|>R_{0}} r^{m}|u|^{2} d x<\infty
$$

$$
\zeta(r)=r^{m / 2}
$$

Then we have

$$
\begin{aligned}
& \int_{|x|>R_{5}}\left\{r^{\max \left\{\delta_{1}, \delta_{2}\right\}} \zeta^{2}+\zeta^{\prime 2}\right\}|u|^{2} d x \\
& =\int_{|x|>R_{5}} r^{m}\left[r^{\max \left\{\delta_{1}, \delta_{2}\right\}}+4^{-1} m^{2} r^{-2}\right]|u|^{2} d x<\infty, \\
& \liminf _{t \rightarrow \infty} \int_{t<|x|<t+1} \zeta^{2}|u|^{2} d x \leq \liminf _{t \rightarrow \infty} t^{-1} \int_{t<|x|<t+1} r^{m+1}|u|^{2} d x=0 .
\end{aligned}
$$

Therefore by Lemma 2.12 we have the assertion.

Lemma 2.15. If

$$
\liminf _{R \rightarrow \infty} R^{\gamma_{1} / 2} \Phi(R) \int_{|x|=R}\left[|\langle A D u, \hat{x}\rangle|^{2}+\left\{r^{-2}+\left(q_{1}\right)_{-}\right\}|u|^{2}\right] d S=0,
$$

then for any constant $m>0$ there exists some constant $R_{7} \geq R_{2}$, where $R_{2}$ is the one given in Lemma 2.8, such that for any $t>s>R_{7}$ we have

$$
e^{m t^{\alpha}} \int_{|x|=t}\langle A \hat{x}, \hat{x}\rangle|u|^{2} d S \leq e^{m s^{\alpha}} \int_{|x|=s}\langle A \hat{x}, \hat{x}\rangle|u|^{2} d S .
$$

Proof. For fixed $m>0$ let

$$
a(r)=2^{-1}\left\{m \alpha r^{\alpha}+n\right\} .
$$

By Lemma 2.6 and (F2) there exists some constant $R_{8} \geq R_{2}$ such that for any $r \geq R_{8}$ we have 


$$
\langle A \hat{x}, \hat{x}\rangle^{-1} r \operatorname{div}(A \hat{x}) \leq n .
$$

Then for any $t>s>R_{8}$ we have by integration by parts

$$
\begin{aligned}
& \left(\int_{|x|=t}-\int_{|x|=s}\right) e^{m r^{\alpha}}\langle A \hat{x}, \hat{x}\rangle|u|^{2} d S \\
& \quad=\int_{s<|x|<t} e^{m r^{\alpha}}\left[2 \operatorname{Re}[\langle A D u, \hat{x}\rangle \bar{u}]+r^{-1}\left\{m \alpha r^{\alpha}+\langle A \hat{x}, \hat{x}\rangle^{-1} r \operatorname{div}(A \hat{x})\right\}\right. \\
& \left.\quad\langle A \hat{x}, \hat{x}\rangle|u|^{2}\right] d x \\
& \quad \leq 2 \int_{s}^{t} e^{m \tau^{\alpha}} d \tau \int_{|x|=\tau}\left[\operatorname{Re}[\langle A D u, \hat{x}\rangle \bar{u}]+a(r) r^{-1}\langle A \hat{x}, \hat{x}\rangle|u|^{2}\right] d S .
\end{aligned}
$$

So we have only to show that there exists some constant $R_{7} \geq R_{8}$ such that for any $\tau \geq R_{7}$ we have

$$
\int_{|x|=\tau}\left[\operatorname{Re}[\langle A D u, \hat{x}\rangle \bar{u}]+a(r) r^{-1}\langle A \hat{x}, \hat{x}\rangle|u|^{2}\right] d S \leq 0 .
$$

Let

$$
\rho(r)=a(\tau) \log r, g(r)=r^{-2 a(\tau)} .
$$

Then by Definition 2.1 and by direct calculation we have

$$
\begin{aligned}
e^{2 \rho(r)} g(r) & =1,2 \rho^{\prime}(r) g(r)+g^{\prime}(r)=0, \\
k_{1}(x)= & -a(\tau)^{2} r^{-2}\langle A \hat{x}, \hat{x}\rangle, k_{2}(x)=-a(\tau) r^{-2}\langle A \hat{x}, \hat{x}\rangle \\
& +a(\tau) r^{-1} \operatorname{div}(A \hat{x}) .
\end{aligned}
$$

Therefore by Lemmas 2.2 and 2.3 and by Definition 2.4 we have for any $t_{1}>\tau$ $>R_{8}$

$$
\begin{aligned}
& \left(\int_{|x|=t_{1}}-\int_{|x|=\tau}\right)\left[\operatorname{Re}[\langle A D u, \hat{x}\rangle \bar{u}]+a(\tau) r^{-1}\langle A \hat{x}, \hat{x}\rangle|u|^{2}\right] d S \\
& =F\left(t_{1} ; a(\tau) \log r, 0, r^{-2 a(\tau)}\right)-F\left(\tau ; a(\tau) \log r, 0, r^{-2 a(\tau)}\right) \\
& =\int_{\tau<|x|<t_{1}} r^{-2 a(\tau)}\left[\langle A D v, \overline{D v}\rangle+\left(q_{1}+\operatorname{Re}\left[q_{2}\right]\right)|v|^{2}\right. \\
& \left.\quad+a(\tau) r^{-2}\{r \operatorname{div}(A \hat{x})-\langle A \hat{x}, \hat{x}\rangle-a(\tau)\langle A \hat{x}, \hat{x}\rangle\}|v|^{2}\right] d x \\
& =-\int_{\tau}^{t_{1}} s_{1}^{-\left\{2 a(\tau)+\left(\gamma_{2} / 2\right)\right\}} \Phi\left(s_{1}\right)^{-1}\left[F\left(s_{1} ; a(\tau) \log r, f_{2}, g_{2}\right)\right. \\
& \left.\quad+2 C_{3} s_{1}^{\left(\gamma_{2} / 2\right)+\beta-2} \Phi\left(s_{1}\right) \sigma\left(s_{1}\right) \int_{|x|=s_{1}}\langle A \hat{x}, \hat{x}\rangle|v|^{2} d S\right] d s_{1}
\end{aligned}
$$




$$
\begin{aligned}
& +\int_{\tau<|x|<t_{1}} r^{-2 a(\tau)}\left[2\langle A \hat{x}, \hat{x}\rangle^{-1}|\langle A D v, \hat{x}\rangle|^{2}+h_{2} r^{-1} \operatorname{Re}[\langle A D v, \hat{x}\rangle \bar{v}]\right. \\
& +\left\{\operatorname{Re}\left[q_{2}\right]+a(\tau) r^{-2}(r \operatorname{div}(A \hat{x})-\langle A \hat{x}, \hat{x}\rangle)\right. \\
& \left.\left.+2 C_{3} r^{\beta-2}\langle A \hat{x}, \hat{x}\rangle \sigma(r)\right\}|v|^{2}\right] d x,
\end{aligned}
$$

where $C_{3}$ is the one given in $(\mathrm{G} 2)$ and $v=v(x ; a(\tau) \log r)$. By $(\mathrm{G} 2)$, Lemma 2.6 and $0<\alpha<\beta$, there exist some constant $C_{12}>0$ and $R_{9} \geq R_{8}$ such that for any $r \geq \tau \geq R_{9}$ we have

$$
\begin{aligned}
2 & \langle A \hat{x}, \hat{x}\rangle^{-1}|\langle A D v, \hat{x}\rangle|^{2}+h_{2} r^{-1} \operatorname{Re}[\langle A D v, \hat{x}\rangle \bar{v}] \\
& +\left\{\operatorname{Re}\left[q_{2}\right]+a(\tau) r^{-2}(r \operatorname{div}(A \hat{x})-\langle A \hat{x}, \hat{x}\rangle)+2 C_{3} r^{\beta-2}\langle A \hat{x}, \hat{x}\rangle \sigma(r)\right\}|v|^{2} \\
\geq & \left\{-8^{-1} h_{2}^{2} r^{-2}\langle A \hat{x}, \hat{x}\rangle-\left(\operatorname{Re}\left[q_{2}\right]\right)_{-}(x)\right. \\
& \left.+a(\tau) r^{-2}(r \operatorname{div}(A \hat{x})-\langle A \hat{x}, \hat{x}\rangle)+2 C_{3} r^{\beta-2}\langle A \hat{x}, \hat{x}\rangle \sigma(r)\right\}|v|^{2} \\
\geq & \left(2^{-1} C_{3} r^{\beta-2} \sigma(r)-C_{12} r^{\alpha-2}\right)|v|^{2} \\
\geq & 0 .
\end{aligned}
$$

By integration by parts we have for any $t_{2} \geq s_{1} \geq R_{8}$

$$
\begin{aligned}
& \left(\int_{|x|=t_{2}}-\int_{|x|=s_{1}}\right) r^{\left(\gamma_{2} / 2\right)+\beta-2} \Phi(r) \sigma(r)\langle A \hat{x}, \hat{x}\rangle|v|^{2} d S \\
& =\int_{s_{1}<|x|<t_{2}} r^{\left(\gamma_{2} / 2\right)+\beta-2} \Phi(r) \sigma(r)[2 \operatorname{Re}[\langle A D v, \hat{x}\rangle \bar{v}] \\
& \quad+r^{-1}\left\{r \operatorname{div}(A \hat{x})+\left(2^{-1}\left(\eta(r)+\sigma(r)+\gamma_{2}\right)\right.\right. \\
& \left.\left.\left.\quad+\beta-2+r \sigma(r)^{-1} \sigma^{\prime}(r)\right)\langle A \hat{x}, \hat{x}\rangle\right\}|v|^{2}\right] d x .
\end{aligned}
$$

Using Lemma 2.5 and the above relation we have for any $t_{2} \geq s_{1} \geq R_{8}$

$$
\begin{aligned}
& F\left(t_{2} ; a(\tau) \log r, f_{2}, g_{2}\right)-F\left(s_{1} ; a(\tau) \log r, f_{2}, g_{2}\right) \\
& \quad+2 C_{3}\left(\int_{|x|=t_{2}}-\int_{|x|=s_{1}}\right) r^{\left(\gamma_{2} / 2\right)+\beta-2} \Phi(r) \sigma(r)\langle A \hat{x}, \hat{x}\rangle|v|^{2} d S \\
& =\int_{s_{1}<|x|<t_{2}} r^{\left(\gamma_{2} / 2\right)-1} \Phi(r)\left[G_{2}(x ; v)+4 a(\tau)\langle A \hat{x}, \hat{x}\rangle^{-1}|\langle A D v, \hat{x}\rangle|^{2}\right. \\
& \quad+2 a(\tau) r^{-1}\left\{h_{2}+r\langle A \hat{x}, \hat{x}\rangle^{-1} \operatorname{div}(A \hat{x})-1\right\} \operatorname{Re}[\langle A D v, \hat{x}\rangle \bar{v}] \\
& \quad+\langle A \hat{x}, \hat{x}\rangle a(\tau) r^{-2}\left[\left\{\gamma_{2}+\eta(r)-2-r\left\langle A D\left(\langle A \hat{x}, \hat{x}\rangle^{-1}\right), \hat{x}\right\rangle\right\} a(\tau)\right. \\
& \left.\quad+h_{2}\left\{r\langle A \hat{x}, \hat{x}\rangle^{-1} \operatorname{div}(A \hat{x})-1\right\}\right]|v|^{2} \\
& +4 C_{3} r^{\beta-1} \sigma(r) \operatorname{Re}[\langle A D v, \hat{x}\rangle \bar{v}] \\
& +2 C_{3} r^{\beta-2}\langle A \hat{x}, \hat{x}\rangle \sigma(r)\left\{r\langle A \hat{x}, \hat{x}\rangle^{-1} \operatorname{div}(A \hat{x})\right.
\end{aligned}
$$




$$
\left.\left.+2^{-1}\left(\eta(r)+\sigma(r)+\gamma_{2}\right)+\beta-2+r \sigma(r)^{-1} \sigma^{\prime}(r)\right\}|v|^{2}\right] d x .
$$

By $\alpha>0$, Lemmas 2.6 and 2.8 there exist some constants $R_{10} \geq R_{9}\left(\geq R_{8} \geq R_{2}\right)$ and $C_{13}>0$ such that for any $r \geq \tau \geq R_{10}$ we have

$$
\begin{aligned}
G_{2}(x ; v) \geq & C_{5} r^{2 \beta-2} \sigma(r)|v|^{2}-C_{6}|\langle A D v, \hat{x}\rangle|^{2}, \\
{\left[2 a(\tau) r^{-1}\right.} & \left.\left\{h_{2}+r\langle A \hat{x}, \hat{x}\rangle^{-1} \operatorname{div}(A \hat{x})-1\right\}+4 C_{3} r^{\beta-1} \sigma(r)\right] \operatorname{Re}[\langle A D v, \hat{x}\rangle \bar{v}] \\
\geq & -a(\tau)\langle A \hat{x}, \hat{x}\rangle^{-1}|\langle A D v, \hat{x}\rangle|^{2}-C_{13}\left(r^{\alpha-2}+r^{2 \beta-2} \sigma(r)^{2} \tau^{-\alpha}\right)|v|^{2}, \\
a(\tau) r^{-2}\langle A \hat{x}, \hat{x}\rangle\left[\left\{\gamma_{2}+\eta(r)-2-r\left\langle A D\left(\langle A \hat{x}, \hat{x}\rangle^{-1}\right), \hat{x}\right\rangle\right\} a(\tau)\right. & \\
& \left.\quad+h_{2}\left\{r\langle A \hat{x}, \hat{x}\rangle^{-1} \operatorname{div}(A \hat{x})-1\right\}\right] \\
\geq- & C_{13} r^{2 \alpha-2}, \\
2 C_{3} r^{\beta-2}\langle & A \hat{x}, \hat{x}\rangle \sigma(r)\left\{r\langle A \hat{x}, \hat{x}\rangle^{-1} \operatorname{div}(A \hat{x})\right. \\
& \left.\quad+2^{-1}\left(\eta(r)+\sigma(r)+\gamma_{2}\right)+\beta-2+r \sigma(r)^{-1} \sigma^{\prime}(r)\right\} \\
\geq- & C_{13}\left(r^{\beta-2}+r^{\beta-1}\left|\sigma^{\prime}(r)\right|\right) .
\end{aligned}
$$

By (F1) $\sim$ (F4) there exists some constant $R_{7} \geq R_{10}$ such that for any $r \geq \tau \geq R_{7}$ we have

$$
\begin{aligned}
& 3 a(\tau)\langle A \hat{x}, \hat{x}\rangle^{-1}-C_{6} \geq 0, \\
& C_{5} r^{2 \beta-2} \sigma(r)-C_{13}\left(r^{\alpha-2}+r^{2 \beta-2} \sigma(r)^{2} \tau^{-\alpha}+r^{2 \alpha-2}+r^{\beta-2}+r^{\beta-1}\left|\sigma^{\prime}(r)\right|\right) \geq 0 .
\end{aligned}
$$

Therefore we have for any $t_{2} \geq s_{1} \geq \tau \geq R_{7}$

$$
\begin{aligned}
& F\left(s_{1} ; a(\tau) \log r, f_{2}, g_{2}\right)+2 C_{3} s_{1}^{\left(\gamma_{2} / 2\right)+\beta-2} \Phi\left(s_{1}\right) \sigma\left(s_{1}\right) \int_{|x|=s_{1}}\langle A \hat{x}, \hat{x}\rangle|v|^{2} d S \\
& \quad \leq F\left(t_{2} ; a(\tau) \log r, f_{2}, g_{2}\right)+2 C_{3} t_{2}^{\left(\gamma_{2} / 2\right)+\beta-2} \Phi\left(t_{2}\right) \sigma\left(t_{2}\right) \int_{|x|=t_{2}}\langle A \hat{x}, \hat{x}\rangle|v|^{2} d S .
\end{aligned}
$$

By (F2), Lemmas 2.2, 2.6, 2.13 and Definition 2.4, for any $\tau \geq R_{7}$ there exist some constants $R_{11} \geq \tau \geq R_{7}$ and $C_{14}>0$ such that for any $t_{2} \geq R_{11}$

$$
\begin{gathered}
F\left(t_{2} ; a(\tau) \log r, f_{2}, g_{2}\right)+2 C_{3} t_{2}^{\left(\gamma_{2} / 2\right)+\beta-2} \Phi\left(t_{2}\right) \sigma\left(t_{2}\right) \int_{|x|=t_{2}}\langle A \hat{x}, \hat{x}\rangle|v|^{2} d S \\
\leq C_{14} t_{2}^{2 a(\tau)+\left(\gamma_{2} / 2\right)+\delta_{3}} \int_{|x|=t_{2}}\left[|\langle A D u, \hat{x}\rangle|^{2}+\left\{r^{\beta-2}+\left(q_{1}\right)_{-}\right\}|u|^{2}\right] d S .
\end{gathered}
$$

Therefore by Lemma 2.14 we have

$$
\begin{aligned}
& \liminf _{t_{2} \rightarrow \infty}\left[F\left(t_{2} ; a(\tau) \log r, f_{2}, g_{2}\right)\right. \\
& \left.\quad+2 C_{3} t_{2}^{\left(\gamma_{2} / 2\right)+\beta-2} \Phi\left(t_{2}\right) \sigma\left(t_{2}\right) \int_{|x|=t_{2}}\langle A \hat{x}, \hat{x}\rangle|v|^{2} d S\right] \leq 0,
\end{aligned}
$$


and then we have for any $s_{1} \geq \tau \geq R_{7}$

$$
F\left(s_{1} ; a(\tau) \log r, f_{2}, g_{2}\right)+2 C_{3} \int_{|x|=s_{1}} r^{\left(\gamma_{2} / 2\right)+\beta-2} \Phi(r) \sigma(r)\langle A \hat{x}, \hat{x}\rangle|v|^{2} d S \leq 0 .
$$

So at last we have for any $t_{1} \geq \tau \geq R_{7}$

$$
\begin{aligned}
& \int_{|x|=\tau}\left[\operatorname{Re}[\langle A D u, \hat{x}\rangle \bar{u}]+a(\tau) r^{-1}\langle A \hat{x}, \hat{x}\rangle|u|^{2}\right] d S \\
& \quad \leq \int_{|x|=t_{1}}\left[\operatorname{Re}[\langle A D u, \hat{x}\rangle \bar{u}]+a(\tau) r^{-1}\langle A \hat{x}, \hat{x}\rangle|u|^{2}\right] d S .
\end{aligned}
$$

Letting $t_{1} \rightarrow \infty$ along a suitable subsequence, we have, by Lemma 2.14 , for any $\tau \geq R_{7}$

$$
\int_{|x|=\tau}\left[\operatorname{Re}[\langle A D u, \hat{x}\rangle \bar{u}]+a(\tau) r^{-1}\langle A \hat{x}, \hat{x}\rangle|u|^{2}\right] d S \leq 0,
$$

which is the desired result.

Lemma 2.16. If

$$
\liminf _{R \rightarrow \infty} R^{\gamma_{1} / 2} \Phi(R) \int_{|x|=R}\left[|\langle A D u, \hat{x}\rangle|^{2}+\left\{r^{-2}+\left(q_{1}\right)_{-}\right\}|u|^{2}\right] d S=0,
$$

then for any constant $m>0$ we have

$$
\int_{|x|>R_{0}} e^{m r^{\alpha}}\left[|D u|^{2}+\left\{1+\left(q_{1}\right)_{-}\right\}|u|^{2}\right] d x<\infty .
$$

Proof. Replacing $m$ with $m+2$ in Lemma 2.15 we have for any $r \geq R_{12}$

$$
\int_{|x|=r}|u|^{2} d S \leq C_{15} e^{-(m+2) r^{\alpha}}
$$

where

$$
\begin{aligned}
& R_{12}=\max \left\{R_{5}, R_{7}\right\}, \\
& C_{15}=C_{1} e^{(m+2) R_{7}^{\alpha}} \int_{|x|=R_{7}}\langle A \hat{x}, \hat{x}\rangle|u|^{2} d S,
\end{aligned}
$$

$R_{5}$ is the one given in Lemma 2.12 and $C_{1}$ is the one given in (A3). So we have

$$
\int_{|x|>R_{12}} e^{(m+1) r^{\alpha}}|u|^{2} d x \leq C_{15} \int_{R_{12}}^{\infty} e^{-r^{\alpha}} d r<\infty .
$$

Let

$$
\zeta(r)=e^{(m / 2) r^{\alpha}}
$$


Then there exists some constant $C_{16}>0$ such that we have

$$
\begin{aligned}
& \int_{|x|>R_{12}}\left\{r^{\max \left\{\delta_{1}, \delta_{2}\right\}} \zeta(r)^{2}+\zeta^{\prime}(r)^{2}\right\}|u|^{2} d x \leq C_{16} \int_{|x|>R_{12}} e^{(m+1) r^{\alpha}}|u|^{2} d x<\infty, \\
& 0 \leq \liminf _{t \rightarrow \infty} \int_{t<|x|<t+1} \zeta(r)^{2}|u|^{2} d x \leq \liminf _{t \rightarrow \infty} e^{-t^{\alpha}} \int_{t<|x|<t+1} e^{(m+1) r^{\alpha}}|u|^{2} d x=0 .
\end{aligned}
$$

Applying Lemma 2.12 we have the assertion.

Now we can prove Theorem 1.1(1).

Proof of Theorem 1.1(1). By Lemma 2.9 we have for any $m \geq 1$ and any $t \geq s \geq R_{3}$

$$
F\left(s ; m r^{\alpha}, f_{2}, g_{2}\right) \leq F\left(t ; m r^{\alpha}, f_{2}, g_{2}\right) .
$$

By Lemma 2.2 and Lemma 2.13 for any $m \geq 1$ there exists some constant $C_{17}$ $>0$ such that for any $t \geq R_{3}$ we have

$$
F\left(t ; m r^{\alpha}, f_{2}, g_{2}\right) \leq C_{17} e^{(2 m+1) r^{\alpha}} \int_{|x|=t}\left[|D u|^{2}+\left\{1+\left(q_{1}\right)_{-}\right\}|u|^{2}\right] d S .
$$

Now we assume that Theorem 1.1(1) is not true. By Lemma 2.16 we have

$$
\liminf _{t \rightarrow \infty} F\left(t ; m r^{\alpha}, f_{2}, g_{2}\right) \leq 0
$$

and then for any $m \geq 1$ and any $s \geq R_{3}$ we have

$$
F\left(s ; m r^{\alpha}, f_{2}, g_{2}\right) \leq 0 .
$$

On the other hand for a fixed $s \geq R_{3}$ we have the followings:

$$
e^{-2 m s^{\alpha}} F\left(s ; m r^{\alpha}, f_{2}, g_{2}\right) \text { is a quadratic in } m \text {, }
$$

the coefficient of $m^{2}$ in $e^{-2 m s^{\alpha}} F\left(s ; m r^{\alpha}, f_{2}, g_{2}\right)$ is

$$
2 \alpha^{2} s^{2 \alpha-2+\left(\gamma_{2} / 2\right)} \Phi(s) \int_{|x|=s}\langle A \hat{x}, \hat{x}\rangle|u|^{2} d S .
$$

Since supp $[u]$ is not a compact set in $\bar{\Omega}$, there exist some constant $R_{13} \geq R_{3}$ such that we have

$$
\int_{|x|=R_{13}}\langle A \hat{x}, \hat{x}\rangle|u|^{2} d S>0 .
$$

Then there exists some constant $m_{0} \geq 1$ such that we have

$$
F\left(R_{13} ; m_{0} r^{\alpha}, f_{2}, g_{2}\right)>0,
$$


which is a contradiction.

In order to prove Theorem 1.1(2) we prepare the following.

Lemma 2.17. Let $0 \leq a<b$ be constants and $v(r)$ be a real-valued function satisfying

$$
\lim _{R \rightarrow \infty} \sup \{|v(r)-v(R)| \mid R+a \leq r \leq R+b\}=0 .
$$

Then for any $\varepsilon^{\prime}>0$ there exists some constant $R_{14} \geq R_{0}$ such that for any $R \geq R_{14}$ we have

$$
\left(b-a-\varepsilon^{\prime}\right) \exp \{v(R)\} \leq \int_{R+a}^{R+b} \exp \{v(r)\} d r \leq\left(b-a+\varepsilon^{\prime}\right) \exp \{v(R)\} .
$$

Proof. For any $\varepsilon^{\prime}>0$ there exists some constant $R_{14} \geq R_{0}$ such that for any $R \geq R_{14}$ and any $r$ satisfying $R+a \leq r \leq R+b$ we have

$$
|\exp \{v(r)-v(R)\}-1|<\varepsilon^{\prime}(b-a)^{-1} .
$$

Then we have for any $R \geq R_{14}$

$$
\left|\int_{R+a}^{R+b}[\exp \{v(r)-v(R)\}-1] d r\right|<\varepsilon^{\prime}
$$

which shows for any $R \geq R_{14}$

$$
-\varepsilon^{\prime} \leq \int_{R+a}^{R+b} \exp \{v(r)-v(R)\} d r-(b-a) \leq \varepsilon^{\prime} .
$$

Now we give the proof of Theorem 1.1(2).

Proof of Theorem 1.1(2). Let $\varepsilon>0$ and let for $r \geq R_{0}$

$$
v(r)=-\left\{2^{-1} \gamma_{1}+\max \left\{0, \delta_{1}, \delta_{2}\right\}\right\} \log r-\frac{1}{2} \int_{R_{0}}^{r} \frac{\eta(r)+\sigma(r)}{r} d r .
$$

Since for any $R \geq R_{0}$ we have

$$
\begin{aligned}
& \sup \{|v(r)-v(R)| \mid R+(\varepsilon / 3) \leq r \leq R+(2 \varepsilon / 3)\} \\
& \quad \leq\left\{\left|\left(\gamma_{1} / 2\right)+\max \left\{0, \delta_{1}, \delta_{2}\right\}\right|+2^{-1} \sup _{r \geq R_{0}}|\eta(r)+\sigma(r)|\right\} \log \frac{R+(2 \varepsilon / 3)}{R}, \\
& \lim _{R \rightarrow \infty} \sup \{|v(r)-v(R)| \mid R+(\varepsilon / 3) \leq r \leq R+(2 \varepsilon / 3)\}=0
\end{aligned}
$$

holds. By Lemma 2.17 with $a=\varepsilon / 3, b=2 \varepsilon / 3$ and $\varepsilon^{\prime}=\varepsilon / 6$, we have for any $R \geq R_{14}$ 


$$
\begin{aligned}
6^{-1} & \varepsilon R^{-\left(\gamma_{1} / 2\right)-\max \left\{0, \delta_{1}, \delta_{2}\right\}} \Phi(R)^{-1} \\
& \leq \int_{R+(\varepsilon / 3)}^{R+(2 \varepsilon / 3)} r^{-\left(\gamma_{1} / 2\right)-\max \left\{0, \delta_{1}, \delta_{2}\right\}} \Phi(r)^{-1} d r \\
& \leq 2^{-1} \varepsilon R^{-\left(\gamma_{1} / 2\right)-\max \left\{0, \delta_{1}, \delta_{2}\right\}} \Phi(R)^{-1} .
\end{aligned}
$$

By Theorem 1.1(1) there exist some constants $R_{15}>R_{0}$ and $C_{18}>0$ such that for any $R \geq R_{15}$

$$
\begin{aligned}
C_{18} R^{-\left(\gamma_{1} / 2\right)} \Phi(R)^{-1} & \leq \int_{|x|=R}\left[|\langle A D u, \hat{x}\rangle|^{2}+\left\{r^{-2}+\left(q_{1}\right)_{-}\right\}|u|^{2}\right] d S \\
& \leq \int_{|x|=R}\left[\langle A \hat{x}, \hat{x}\rangle\langle A D u, \overline{D u}\rangle+\left\{r^{-2}+\left(q_{1}\right)_{-}\right\}|u|^{2}\right] d S \\
& \leq C_{1}^{2} \int_{|x|=R}\left[|D u|^{2}+\left\{r^{-2}+\left(q_{1}\right)_{-}\right\}|u|^{2}\right] d S,
\end{aligned}
$$

where $C_{1} \geq 1$ is the one given in (A3). Let $\xi_{R}(r) \in C_{0}^{1}(R, R+\varepsilon)$ satisfy the following: $\xi_{R}(r)=1$ for $R+(\varepsilon / 3) \leq r \leq R+(2 \varepsilon / 3), 0 \leq \xi_{R}(r) \leq 1$ for $R \leq r \leq R$ $+\varepsilon$ and there exists some constant $C_{19}>0$ such that for any $R \geq R_{0}$ and any $r \geq R \quad$ we have $\left|\xi_{R}^{\prime}(r)\right| \leq C_{19}$. Applying Lemma 2.11 with $\psi(r)$ $=\xi_{R}(r) r^{-\frac{1}{2} \max \left\{0, \delta_{1}, \delta_{2}\right\}}$, we have for any $R \geq \max \left\{R_{5}, R_{14}, R_{15}\right\}$

$$
\begin{aligned}
& 6^{-1} \varepsilon C_{18} R^{-\left(\gamma_{1} / 2\right)-\max \left\{0, \delta_{1}, \delta_{2}\right\}} \Phi(R)^{-1} \\
& \leq \int_{R+(\varepsilon / 3)}^{R+(2 \varepsilon / 3)} C_{18} r^{-\left(\gamma_{1} / 2\right)-\max \left\{0, \delta_{1}, \delta_{2}\right\}} \Phi(r)^{-1} d r \\
& \leq C_{1}^{2} \int_{R<|x|<R+\varepsilon}\left(\xi_{R}(r) r^{-\frac{1}{2} \max \left\{0, \delta_{1}, \delta_{2}\right\}}\right)^{2}\left\{|D u|^{2}+\left(q_{1}\right)_{-}\right\}|u|^{2} d x \\
& +C_{1}^{2} \int_{R<|x|<R+\varepsilon} r^{-\max \left\{0, \delta_{1}, \delta_{2}\right\}-2}|u|^{2} d x \\
& \leq C_{1}^{2} C_{8} \int_{R<|x|<R+\varepsilon}\left[1+2\left\{\xi_{R}^{\prime}(r)^{2}+4^{-1}\left(\max \left\{0, \delta_{1}, \delta_{2}\right\}\right)^{2}\right\}\right]|u|^{2} d x \\
& +C_{1}^{2} \int_{R<|x|<R+\varepsilon}|u|^{2} d x \\
& \leq C_{20} \int_{R<|x|<R+\varepsilon}|u|^{2} d x,
\end{aligned}
$$

where

$$
C_{20}=C_{1}^{2}\left[C_{8}\left\{1+2 C_{19}^{2}+2^{-1}\left(\max \left\{0, \delta_{1}, \delta_{2}\right\}\right)^{2}\right\}+1\right]>0 .
$$

This shows the assertion. 
Lastly we give the proof of Theorem 1.1(3).

Proof of Theorem 1.1(3). By Theorem 1.1(2) with $\varepsilon=1$ there exist some integer $N_{0} \geq R_{0}$ and some constant $C_{21}>0$ such that for any integer $N \geq N_{0}$ we have

$$
\int_{N<|x|<N+1}|u|^{2} d x \geq C_{21} N^{-\left(\gamma_{1} / 2\right)-\max \left\{0, \delta_{1}, \delta_{2}\right\}} \Phi(N)^{-1} .
$$

Applying Lemma 2.17 with $a=0, b=1$ and $\varepsilon^{\prime}=1$, there exists some integer $N_{1} \geq R_{0}$ such that for any integer $N \geq N_{1}$ we have

$$
2 N^{-\left(\gamma_{1} / 2\right)-\max \left\{0, \delta_{1}, \delta_{2}\right\}} \Phi(N)^{-1} \geq \int_{N}^{N+1} r^{-\left(\gamma_{1} / 2\right)-\max \left\{0, \delta_{1}, \delta_{2}\right\}} \Phi(r)^{-1} d r .
$$

Let $N_{2}=\max \left\{N_{0}, N_{1}\right\}$. Then for any integer $M>0$ we have

$$
\begin{aligned}
\int_{N_{2}}^{N_{2}+M}|u|^{2} d x & \geq C_{21} \sum_{n=N_{2}}^{N_{2}+M-1} n^{-\left(\gamma_{1} / 2\right)-\max \left\{0, \delta_{1}, \delta_{2}\right\}} \Phi(n)^{-1} \\
& \geq 2^{-1} C_{21} \int_{N_{2}}^{N_{2}+M} r^{-\left(\gamma_{1} / 2\right)-\max \left\{0, \delta_{1}, \delta_{2}\right\}} \Phi(r)^{-1} d r
\end{aligned}
$$

which shows the assertion.

\section{§3. Proof of Theorems $\mathbf{1 . 2}$ and $\mathbf{1 . 3}$}

Proof of Theorem 1.2. By Lemma 4.1 of Uchiyama [3], Lemma 2.3 is also true under our weak condition (C3)'. So we can follow the proof of Theorem 1.1 .

Proof of Theorem 1.3. Lemma 2.3 also holds under our weak condition (C3)'. By Definition 2.4 we have

$$
\begin{aligned}
G_{i}(x ; w)= & \sigma(r)\left|\partial_{r} w\right|^{2}+\left(2-\gamma_{i}-\eta(r)\right)\left\{|\nabla w|^{2}-\left|\partial_{r} w\right|^{2}\right\}+2 r \operatorname{Re}\left[\overline{q_{2} w} \partial_{r} w\right] \\
& +\operatorname{Re}\left[\left\langle\nabla w, \nabla h_{i}\right\rangle \bar{w}\right]+(2 r)^{-1} h_{i}\left\{\eta(r)+\sigma(r)-\gamma_{i}-2\right\} \operatorname{Re}\left[\bar{w} \partial_{r} w\right] \\
& -\left\{r \partial_{r} q_{1}(x)+\left(\gamma_{i}+\eta(r)\right) q_{1}(x)-h_{i} \operatorname{Re}\left[q_{2}\right]\right\}|w|^{2},
\end{aligned}
$$

where

$$
h_{i}(x)=n-1+2^{-1}\left\{\sigma(r)-\eta(r)-\gamma_{i}\right\} .
$$

Since $h_{i}$ is a function depending only on $r$, we have

$$
\operatorname{Re}\left[\left\langle\nabla w, \nabla h_{i}\right\rangle \bar{w}\right]=h_{i}^{\prime}(r) \operatorname{Re}\left[\bar{w} \partial_{r} w\right] .
$$

So in the estimination of $G_{i}(x ; w)$ given in Lemma 2.7 and Lemma 2.8 , we need 
not use the term $\left(2-\gamma_{i}-\eta(r)\right)\left\{|\nabla w|^{2}-\left|\partial_{r} w\right|^{2}\right\}$, which is non-negative by our weak condition (F2)'. Therefore Lemmas 2.7 and 2.8 are also true and we can follow the proof of Theorem 1.1.

\section{References}

[1] Agmon, S., Lower bounds for solutions of Schrödinger equations, J. Analyse Math. $23(1970), 1-25$.

[2] Eastham, M.S.P. and Kalf, H., Schrödinger-type operators with continuous spectra, Research Notes in Mathematics 65, Pitman Advanced Publishing Program, 1982.

[3] Uchiyama, J., Polynomial growth or decay of eigenfunctions of second-order elliptic operators, Publ. RIMS, Kyoto Univ., 23(1987), 975-1006.

[4] Yamada, O., A note on the non-existence of eigenvalues of Schrödinger equations, Memoirs of the Research Institute of Science and Engineering, Ritsumeikan Univ., 32(1977), 1-6. 
